\title{
Multi-Fidelity Approach to Dynamics Model Calibration
}

\author{
Ghina N Absi ${ }^{\mathrm{a}}$, Sankaran Mahadevan ${ }^{\mathrm{b}^{*}}$
}

${ }^{a}$ Graduate Student, Department of Civil and Environmental Engineering, Vanderbilt University, 2301 Vanderbilt Place, Nashville, TN, 37235, USA

b John R. Murray Sr. Professor, Department of Civil and Environmental Engineering, Vanderbilt University, 2301 Vanderbilt Place, Nashville, TN, 37235, USA

\section{ABSTRACT}

This paper investigates the use of structural dynamics computational models with multiple levels of fidelity in the calibration of system parameters. Different types of models may be available for the estimation of unmeasured system properties, with different levels of physics fidelity, mesh resolution and boundary condition assumptions. In order to infer these system properties, Bayesian calibration uses information from multiple sources (including experimental data and prior knowledge), and comprehensively quantifies the uncertainty in the calibration parameters. Estimating the posteriors is done using Markov Chain Monte Carlo sampling, which requires a large number of computations, thus making the use of a high-fidelity model for calibration prohibitively expensive. On the other hand, use of a low-fidelity model could lead to significant error in calibration and prediction. Therefore, this paper develops an approach for model parameter calibration with a low-fidelity model corrected using higher fidelity simulations, and investigates the trade-off between accuracy and computational effort. The methodology is illustrated for a curved panel located in the vicinity of a hypersonic aircraft engine, subjected to acoustic loading. Two models (a frequency response analysis and a full time history analysis) are combined to calibrate the damping characteristics of the panel.

\footnotetext{
* Corresponding author. Tel.: + 1615322 3040; fax: + 16153223365.

E-mail address: Sankaran.mahadevan@vanderbilt.edu
} 


\section{KEYWORDS}

Multi-fidelity, Bayesian Calibration, Hypersonic Vehicle, Model Uncertainty, Information Fusion, Damping Coefficient

\section{INTRODUCTION}

With the continuous development of faster and more efficient computational capabilities, higher fidelity computer simulations are increasingly being used to try to predict the behavior of systems. Specifically in dynamics problems, being able to forecast the response of a structure over time is an important need. High fidelity dynamic simulation allows the prediction of performance not only under normal operating conditions but also during startup, shutdown and abnormal conditions, especially when behavior is highly non-linear, and linear low fidelity models are inaccurate [1]. In hypersonic aircraft simulations for example, Candler et al. [2] [3], Higgins and Schmidt [4], and many others have investigated high fidelity analyses. Considerable effort has also been reported in developing reduced-order models that are cheaper and faster to run [5] [6] [7]. These high fidelity simulations, as well as the reduced-order models, are useful only when they are good representations of the actual structure and the underlying physics that cause the observed behaviors are taken into account [8].

In order to obtain a model that is structurally equivalent to an experimental setup, one necessary step is model calibration: quantifying the errors and adjusting the unknown model parameters to minimize the difference between the model output and the experimental data. Errors arise in the numerical methods used to solve the problem, but also in the limitations of the available experimental data. Christie et al. [9] argue that knowledge about the state of a complex phenomenon system and the governing physical processes is often limited and/or inaccurate, and 
propose building error models. These error models aim to provide an independent estimate of the known shortcomings of the simulations, and define quantitative bounds of the possible errors. However, they are not necessarily used to increase the accuracy of the simulation.

Uncertainty in simulations has multiple sources:

(1) Natural variability (aleatoric uncertainty) which is not reducible, but generally modeled by assigning probability distributions to the variables.

(2) Statistical uncertainty (reducible, epistemic uncertainty). It arises from sparse and/or imprecise data.

(3) Model uncertainty (reducible, epistemic uncertainty), which is due to uncertainty in model parameters, model form error, and solution approximations [10]. The solution approximation errors arise due to reduced order models, surrogate models, discretization errors, truncation and round off.

Mottershead and Friswell [11] offer a similar grouping of modeling errors into three types: (a) model form errors (due to assumptions regarding the underlying physics of the problem, especially with strongly nonlinear behavior), (b) model parameter uncertainty (due to assumptions regarding boundary conditions, parameter distributions and simplifying assumptions), and (c) model order errors (arising from the discretization of complex geometry and loading). Liang and Mahadevan [12] proposed a systematic methodology to quantify various error and uncertainty sources in model prediction.

Three types of modeling approaches have been pursued in structural dynamics for complicated mechanical systems: (1) finite element models, (2) reduced order models and (3) surrogate models. Finite element analysis (FEA) is commonly used in the dynamics modeling of engineering structures with complicated geometry and under complex loading conditions. 
Construction of the FEA model incorporates many assumptions by the analyst about the system properties and excitation. Two principal qualities are desired in a functional finite element model of structural dynamics [13]: (1) physical significance, i.e., the model should properly represent how the mass, stiffness and damping are distributed, and (2) correctness, i.e., the observations from dynamics experiments are accurately predicted by the model. High-fidelity dynamic finite element analysis of complex mechanical systems is quite expensive, and considerable research has been done to construct cheaper and simpler surrogate models, equivalent static models, or reduced-order models. However, the errors and uncertainties in calibration and prediction increase with the reduction in model fidelity. Computationally efficient models have to be cheap enough to allow multiple repetitions of the simulations, but also retain precious information available from rigorous but more expensive models.

Many studies have concentrated on developing reduced-order models (ROM) to replace full fidelity dynamic analyses. McEwan et al [14] [15] proposed the Implicit Condensation (IC) method that included the non-linear terms of the equation of motion, but restricted the nonlinear function to cubic stiffness terms. The IC method can only predict the displacements covered by the bending modes. Other methods explicitly include additional equations to calculate the membrane displacements in the ROM, such as those by Rizzi et al. [16] [17] and Mignolet et al. [18] [19]. The effectiveness of these reduced order models is directly related to their ability to predict the trends in higher fidelity models and/or experimental data [20].

Several studies have replaced the expensive computational model with surrogate models such as Polynomial Chaos Expansion (PCE), Gaussian process models, etc. This approach facilitates running inexpensive simulations. Dynamic finite element models are particularly 
expensive to run, and Hemez and al. [21] also used a polynomial surrogate to reduce the computational cost in calibrating the parameters of a transient dynamics problem.

Different methodologies have also been presented to deal with multi-fidelity surrogate modeling, where codes and/or models of different complexities are available. Haftka [22] and Hutchison et al. [23] calculated a high to low fidelity ratio, and applied it as a scaling factor to the low fidelity data to refine the low fidelity surrogate model. Kennedy and O’Hagan [24] developed an autoregressive approach to combine surrogate models of different fidelities. Leary et al. [25] use the difference between the high fidelity and low fidelity data at certain locations to train artificial neural networks and kriging interpolation. Forrester and al. [26] considered partially converged simulations from a high fidelity model as low fidelity data.

In order to improve the performance of simulation models, model calibration, commonly known as model updating in dynamics literature, has been extensively investigated. The distance between the measured data and the model prediction is computed. The goal is to minimize this distance (using least squares, for example) by varying the design parameters. Direct updating methods have been proposed by computing closed-form solutions for the global stiffness and mass matrices using the structural equations of motion [27] [28]. The generated matrices are faithful to modal analyses, but do not always maintain structural connectivity, and may not retain physical significance. Other iterative methods study the changes in model parameterization to evaluate the type and the location of the erroneous parameters, and vary these parameters in an effort to minimize the difference between the experimental data and the FE model predictions [29]. Datta et al. [30] used complex mode data to update FEA models with validated models and synthetic data, however performing complex mode identification tests is difficult. Other studies propose updating different parameters separately, in a two-step method. Arora et al. [31] update 
mass and stiffness matrices in the first step, and then use the updated results in the second step to update the damping matrix. Yuan and Yu [32] claim that the difference in scale between the parameters (elastic moduli and hysteresis damping in this case) degrades the results if the updating is done in one step. These methods are also only valid if the parameters are not correlated.

Three approaches are commonly available for calibration of model parameters with input-output data: least squares, maximum likelihood, and Bayesian calibration. Bayesian calibration is able to combine both prior (subjective) information and experimental data, and is able to quantify the epistemic uncertainty in the calibration result. Kennedy and O'Hagan [33] have developed a Bayesian calibration framework (commonly known as $\mathrm{KOH}$ framework), which includes multiple sources of uncertainty, specifically a discrepancy function between the model and the experimental data. Including appropriate model discrepancy functions has been shown to improve calibration of model parameters [34]. Simoen et al. [35] argue that calibration is extremely sensitive to small errors, and that an inadequate quantification of uncertainty may weaken robust inverse methodologies. They discuss that systematic errors influence the likelihood function, and special care should be taken in analyzing the error sources (using available experimental data), and including their correlations in the likelihood expression. Further, Goulet [36] shows that when using Bayesian inference, the assumption of independent uncertainties may bias the posterior $p d f$.

Bayesian model updating methods have also been developed for dynamics problems specifically. Beck and Katafygiotis [37] developed a Bayesian probabilistic system identification framework, in which the structural model and the probability models used to quantify the uncertainties are updated using experimental observations, and the output is a combination of the 
optimum solutions of the individual probability models. Yuen and Katafygiotis [38] then presented a Bayesian time-domain methodology for model calibration of linear MDOF systems that updates the modal parameters using one set of observed data, without the need to calculate multiple optima. It is based on an appropriately selected multi-variate Gaussian distribution for modal parameters. On the other hand, Yuen and Katafygiotis [39] and Au [40] make use of models in the frequency domain, and $\mathrm{Au}$ [40] calibrates modal parameters using well separated modes and a more general case of multiple closely spaced modes [41]. These approaches focus on the updating of modal parameters, and the development of analytical solutions for the Bayesian updating, especially the likelihood function. The class of problems of interest in this paper is different, i.e., problems with complicated excitation, and non-linear structural behavior (e.g., excitation-dependent damping), that require finite element-based numerical solutions. Furthermore, analytical solutions for Bayesian updating are not available, and numerical approaches such as Markov Chain Monte Carlo sampling need to be pursued instead.

In a general situation where conjugate distributions cannot be assumed or analytical solutions are not available, Bayesian model calibration uses Markov Chain Monte Carlo (MCMC) sampling which requires thousands of samples. The expense is further increased if the number of parameters is high, or the model output is far from the experimental data; therefore, the original simulation model may be replaced by an inexpensive surrogate model to reduce the computational cost. However, building a surrogate model also requires training points, which are generated by running the original model at a certain number of input conditions. Obviously, the larger the number of training points, the more accurate is the surrogate model. If the original high-fidelity model is expensive, this may limit the number of training points. On the other hand, if a lower fidelity model is available, this may help to generate a large number of training points, 
but the accuracy of the resulting surrogate model would be questionable. Such a scenario motivates the need for a methodology that can efficiently combine models of lower and higher fidelity to balance accuracy vs. computational effort in the Bayesian calibration of system model parameters.

The aim of this study is to develop a novel approach of fusing information from models of different levels of fidelity in the Bayesian calibration of the model parameters. In order to balance computational effort and accuracy, the proposed method uses a corrected low-fidelity surrogate model. First, it takes advantage of the information available in high-fidelity simulation to improve the low-fidelity surrogate model, and then uses the improved low-fidelity surrogate model for calibration. Use of the low-fidelity surrogate model facilitates computational efficiency. Furthermore, the improvement with high-fidelity results before calibration with experimental data provides a stronger, physics-informed prior for the calibration parameters. This takes into account additional high-fidelity information that may be missing in the lowfidelity model (such as non-linearity, additional variables, etc.). In the same way, information available in the low-fidelity model and missing from the high-fidelity model is also retained, such as modeling the full time history vs. a small segment of the time history (the high-fidelity model may be too expensive to run for the full time history). The improved low-fidelity surrogate model is inexpensive to use in calibration, and yet becomes more accurate. This is particularly useful when limited experimental data are available, and a reliable, nevertheless fast model is needed for calibration.

Section 2 briefly reviews the basic concepts and methods used in the calibration of damping in structural dynamics, and in the Bayesian calibration process. Section 3 presents the proposed multi-fidelity calibration methodology in detail. Section 4 illustrates the proposed 
methodology using an application problem of a curved panel subjected to acoustic loading. Section 5 summarizes the paper's contributions and possible future extensions.

\section{Background}

The approach developed in this paper for combining models of multiple fidelity for model parameter calibration is quite general and is applicable to a wide variety of physics models. However, this paper focuses on structural dynamics problems to develop this approach, in particular the calibration of damping parameters in a structural dynamics model.

\subsection{Calibration of damping}

Consider the structural dynamics model:

$$
[\boldsymbol{M}]\{\ddot{x}(t)\}+[\boldsymbol{C}]\{\dot{x}(t)\}+[\boldsymbol{K}]\{x(t)\}=\{f(t)\}
$$

where $[\mathbf{M}]$ is the mass matrix, $[\mathbf{K}]$ is the stiffness matrix and $[\mathbf{C}]$ is the damping matrix. The mass matrix could be calculated based on measurements of smaller components of the structure, or based on information about material density and component and system geometry. Similarly, the stiffness matric could be calculated based on known or measured mechanical properties (e.g., Young's modulus) and the geometry of the components and system. However, damping cannot be directly calculated or measured; it has to be inferred from the dynamic response of the structure under some excitation.

By definition, damping is the dissipation of energy from a vibrating structure, and is dependent on the active physical mechanisms in the structure. These mechanisms are very complicated, and not precisely understood. The types of damping present depend on which mechanism dominates in a given structural configuration [42]. For example, structural (or hysteretic) damping is a property that is intrinsic to the material. Coulomb damping (also known as frictional damping) is used to represent dry friction in sliding surfaces, such as structure joints. Non-viscous damping 
models are such that the damping forces depend on the structural response history. In multiple degree-of-freedom systems, the most popular approach is to assume viscous damping, which is a special case of the general linear damping model that has no memory.

Often, viscous damping has been assumed and derived from the shape of the frequency response curve in several ways [43]. One way is by using the half-power bandwidth method (Fig. 1), in which the viscous damping ratio $(\zeta)$ is determined from the frequencies for which the power input is half the input at resonance.

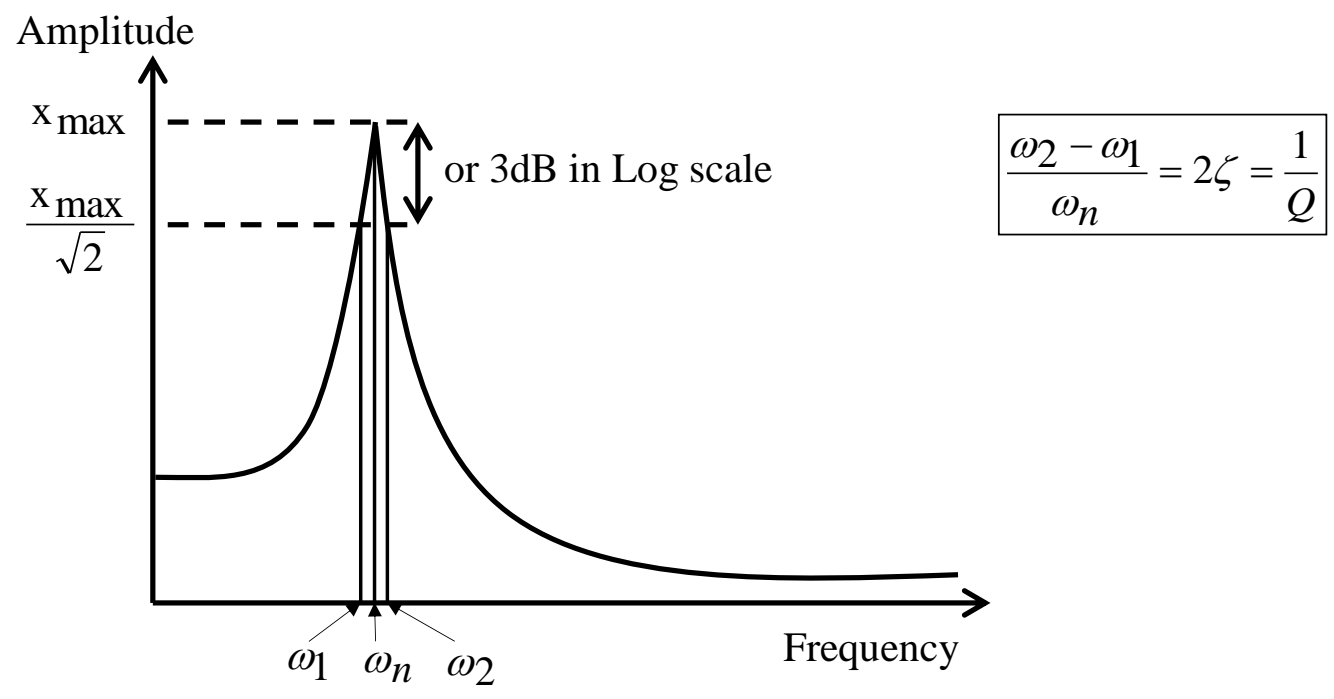

Fig. 1: Half-power bandwidth method

Another method is by conducting experiments at resonance to measure the phase relationship between the input force and the resulting displacements, without having to construct the frequency response curve. The applied load is exactly balanced by the damping force when resonance is established by adjusting the input frequency until the response is $90^{\circ}$ out of phase with the load. For one given loading cycle, the damping force displacement diagram can be 
plotted (Fig. 2), and the damping coefficient determined directly as the ratio of the maximum damping force $\left(f_{D, \max }\right)$ to the maximum velocity $\left(\dot{v}_{\max }\right)$. Linear damping results in an elliptic shape (dashed line), and the damping coefficient ( $\zeta$ is directly derived as shown in Fig. 2. If the damping is nonlinear (solid line), an ellipse with the same area as the original shape is constructed, and an equivalent viscous damping is then defined as that causing the same energy loss per cycle as in the observed force-displacement diagram.

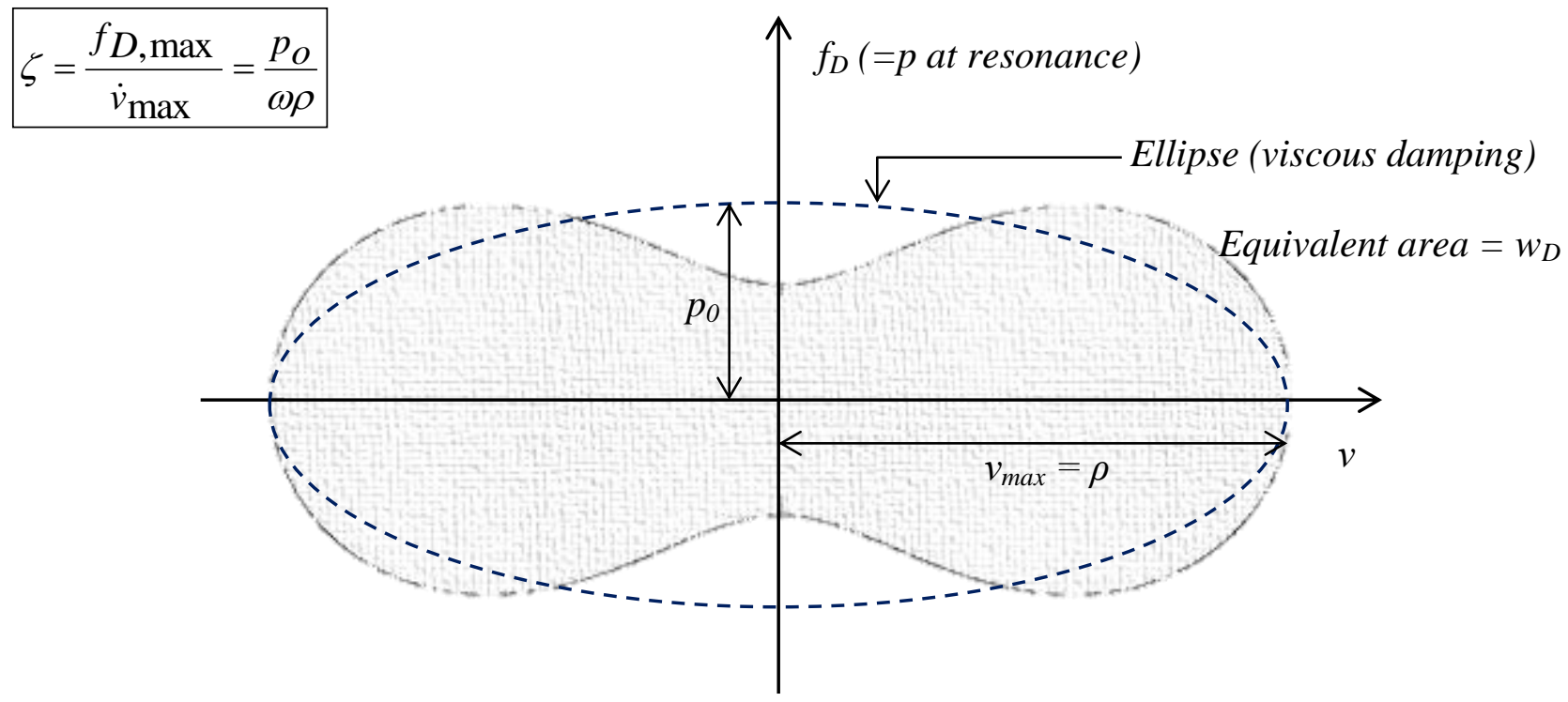

Fig. 2: Actual and equivalent damping energy per cycle

\subsection{Bayesian Calibration}

Bayesian calibration of model parameters may be expressed as

$$
f_{\boldsymbol{\Theta}}\left(\boldsymbol{\Theta} \mid \boldsymbol{Y}_{\text {obs }}\right) \propto f_{\boldsymbol{\Theta}}(\boldsymbol{\Theta}) \cdot L(\boldsymbol{\Theta})
$$


where $f_{\boldsymbol{\Theta}}\left(\boldsymbol{\Theta} \mid \boldsymbol{Y}_{\boldsymbol{o b s}}\right)$ is the posterior distribution of the parameter $\boldsymbol{\Theta}$ after calibration using the data $\boldsymbol{Y}_{\boldsymbol{o b s}}, f_{\boldsymbol{\Theta}}(\boldsymbol{\Theta})$ is the prior distribution of $\boldsymbol{\Theta}$ (assumed by the analyst), and $L(\boldsymbol{\Theta})$ is the likelihood function (i.e., probability of observing the data $\boldsymbol{Y}_{\boldsymbol{o b s}}$, given a value of the calibration parameter). The prior and posterior densities represent the analyst's epistemic uncertainty about the calibration parameter; increased availability of data in general leads to reduction in the variance of the posterior density, thus indicating increasing knowledge or certainty about the calibration parameter. Samples of the posterior can be constructed using a Markov Chain Monte Carlo (MCMC) algorithm. Several algorithms are available for MCMC sampling: MetropolisHastings [44], Gibbs sampling [45], slice sampling [46], etc. Slice sampling is used in the numerical example in this paper to evaluate Eq. (2). It is based on the observation that to sample a random variable, one can sample uniformly from the area under the PDF. The simplest implementation (for a uni-variate distribution without the need to reject any points) consists of first sampling a random value $y$ between zero and the maximum PDF value $y_{\max }$, and then sampling $x$ from the slice under the PDF, as shown in Fig. 3.

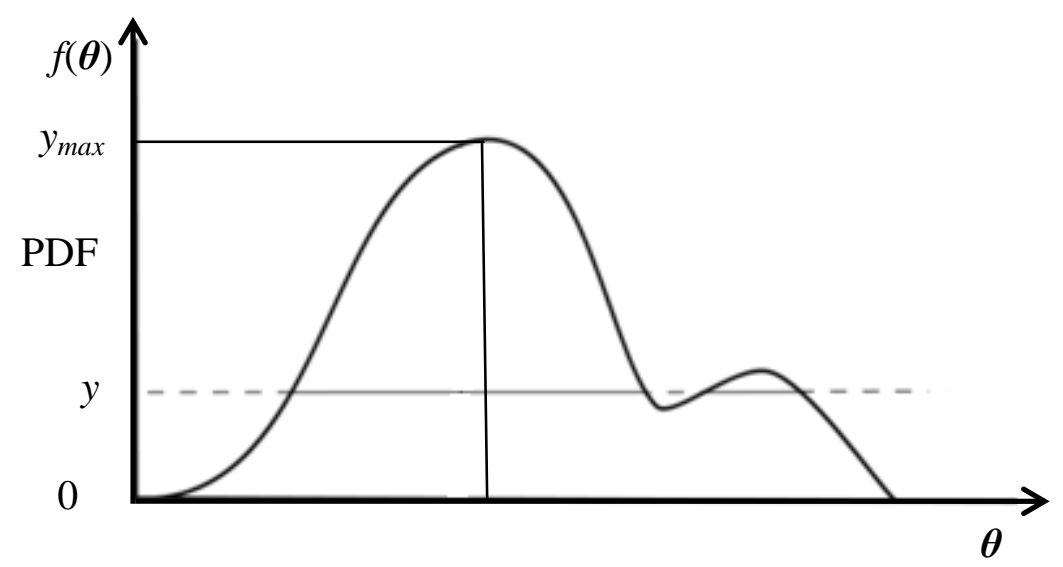

Fig. 3: Simple implementation of slice sampling 
Slice sampling requires many simulations of the model being used for calibration. Highfidelity physics models are very expensive to run in calibration, thus we replace them with inexpensive surrogate models.

\subsection{Surrogate Model - Polynomial Chaos Expansion}

Many surrogate modeling techniques have been developed in the literature, such as linear/quadratic polynomial-based response surfaces [47], artificial neural networks [48], support vector machines (SVM) [49], polynomial chaos expansion (PCE) [50], and Gaussian process (GP) interpolation (or Kriging) [51]. In this paper, PCE is used for the sake of illustration, to replace the original FEA model for inexpensive sampling during the calibration process. PCE is a regression-based surrogate model that represents the output of a model with a series expansion in terms of standard random variables (SRVs). Consider a model $\boldsymbol{y}=f(\boldsymbol{x})$ where $\boldsymbol{x}=\left\{x_{1}, x_{2}, \ldots\right.$, $\left.x_{k}\right\}^{T}$ is a vector of input random variables. We construct a PCE model to replace $f(\boldsymbol{x})$ using $n$ multi-dimensional Hermite polynomials as basis functions:

$$
\boldsymbol{y}=\sum_{j=0}^{n} \theta_{j} \phi_{j}(\boldsymbol{\xi})=\boldsymbol{\theta}^{T}(\boldsymbol{\xi})+\boldsymbol{\varepsilon}_{\text {surr }}
$$

where $\xi$ is a vector of independent standard normal random variables which correspond to the original input $\boldsymbol{x}[52] . \varphi()=.\left\{\phi_{0}(.), \phi_{1}(.), \ldots, \phi_{n}(.)\right\}^{T}$ are the Hermite polynomial basis functions, and $\boldsymbol{\theta}=\left\{\theta_{0}, \theta_{1}, \ldots, \theta_{n},\right\}^{T}$ are the corresponding coefficients that can be estimated by the least squares method. A collocation point method can be used to efficiently select training points where the original model is evaluated [53]. Suppose that $m$ training points $\left(\xi_{i}, y_{i}\right), i=1,2, \ldots, m$ are available. Under the Gauss-Markov assumption [54], the surrogate model error $\boldsymbol{\varepsilon}_{\text {surr }}$ asymptotically follows a normal distribution with zero mean and variance given by

$$
\operatorname{Var}\left[\boldsymbol{\varepsilon}_{\text {surr }}\right] \approx s^{2}+s^{2} \varphi(\boldsymbol{\xi})^{T}\left(\boldsymbol{\Phi}^{T} \boldsymbol{\Phi}\right)^{-1} \varphi(\boldsymbol{\xi})
$$


where

$\boldsymbol{\Phi}=\left\{\varphi\left(\xi_{1}\right), \varphi\left(\xi_{2}\right), \ldots, \varphi\left(\xi_{m}\right)\right\}^{T}$ and $s^{2}=\frac{1}{m-n} \sum_{i=1}^{m}\left[y_{i}-\boldsymbol{\theta}^{T} \varphi\left(\xi_{i}\right)\right]^{2}$

In all calibration calculations in this paper, the original finite element models are replaced with PCEs; the surrogate model error is calculated and added to the output by randomly sampling a value from its distribution.

\section{MULTI-FIDELITY CALIBRATION METHOD}

In this section, the concept of calibration is extended from a simple calibration using experimental data with a single model, to a two-step approach, by combining models of different fidelities. Assume that we have two models $G_{l}(\boldsymbol{X}, \boldsymbol{\theta})$ and $G_{2}(\boldsymbol{X}, \boldsymbol{\theta})$ where $G_{2}(\boldsymbol{X}, \boldsymbol{\theta})$ has higher fidelity than $G_{l}(\boldsymbol{X}, \boldsymbol{\theta})$. Even $G_{l}(\boldsymbol{X}, \boldsymbol{\theta})$ is expensive to use in Bayesian calibration, which requires thousands of MCMC samples, therefore the low fidelity model is replaced by a fast running surrogate model $S_{l}(\boldsymbol{X}, \boldsymbol{\theta})$ in the proposed methodology. In order to build this surrogate model, the model $G_{l}(X, \theta)$ needs to be evaluated multiple times. Model $G_{2}(X, \theta)$ is also run a small number of times to get high fidelity data. Assuming higher fidelity models run much slower than lower fidelity ones, time constraints will allow far less high fidelity simulations. In the proposed methodology, we do not replace $G_{2}(X, \theta)$ with a surrogate model. The results of $G_{2}(X, \theta)$ runs are only used to correct or adjust the low-fidelity model.

\subsection{Model Calibration under Uncertainty}

The experimental observation $\boldsymbol{Y}_{\boldsymbol{o b s}}$ is expressed in terms of the input $\boldsymbol{X}$, parameters $\boldsymbol{\theta}$, the errors and the model output as follows:

$$
Y_{o b s}+\varepsilon_{o b s}=G\left(X+\varepsilon_{i n}, \theta\right)+\varepsilon_{s u r r}+\varepsilon_{d}
$$

where $\varepsilon_{i n}$ : vector of input measurement errors, one for each input measured 
$\varepsilon_{o b s}$ : vector of measurement errors for the multiple outputs

$\boldsymbol{\varepsilon}_{\boldsymbol{d}}$ : vector of model discrepancy terms (discrepancy between prediction and observation), one for each output

$\boldsymbol{\varepsilon}_{\text {surr }}$ : vector of surrogate model errors, one for each output

The system parameters $\boldsymbol{\theta}$ are calibrated using Bayes' theorem as:

$$
\begin{aligned}
& \pi\left(\theta, \sigma_{o b s}, \varepsilon_{d} \mid y_{D}\right)=\frac{L\left(\theta, \sigma_{o b s}, \varepsilon_{d}\right) \pi\left(\theta, \sigma_{o b s}, \varepsilon_{d}\right)}{\int L\left(\theta, \sigma_{o b s}, \varepsilon_{d}\right) \pi\left(\theta, \sigma_{o b s}, \varepsilon_{d}\right) d \theta d \sigma_{o b s} d \varepsilon_{d}} \\
& L\left(\theta, \sigma_{o b s}, \varepsilon_{d}\right) \propto \prod_{i=1}^{m} \pi\left(Y_{o b s i}=y_{D i} \mid x_{D i}, \theta, \sigma_{o b s}, \varepsilon_{d}\right)
\end{aligned}
$$

where $\pi(*)$ denotes the joint probability density function (PDF) of the variables, and $\pi\left(\boldsymbol{\theta}, \boldsymbol{\sigma}_{\boldsymbol{o b s}}, \boldsymbol{\varepsilon}_{\boldsymbol{d}}\right)$ is the prior joint PDF of $\boldsymbol{\theta}, \boldsymbol{\sigma}_{\boldsymbol{o} b s}$ and $\varepsilon_{d} ; \pi\left(\boldsymbol{\theta}, \boldsymbol{\sigma}_{\boldsymbol{o b s}}, \boldsymbol{\varepsilon}_{\boldsymbol{d}} \mid \boldsymbol{y}_{\boldsymbol{D}}\right)$ is the joint posterior PDF of $\boldsymbol{\theta}, \boldsymbol{\sigma}_{\boldsymbol{o} \boldsymbol{s}}$ and $\boldsymbol{\varepsilon}_{\boldsymbol{d}}$ given $\boldsymbol{y}_{\boldsymbol{D}}$; and $L\left(\boldsymbol{\theta}, \boldsymbol{\sigma}_{\boldsymbol{o} \boldsymbol{b} s}, \boldsymbol{\varepsilon}_{\boldsymbol{d}}\right)$ is the joint likelihood function of $\boldsymbol{\theta}, \boldsymbol{\sigma}_{\boldsymbol{o b s}}, \boldsymbol{\varepsilon}_{\boldsymbol{d}}$. $\boldsymbol{y}_{\boldsymbol{D} i}$ is the vector of the $n$ outputs $\boldsymbol{Y}_{\boldsymbol{o b s i}}$ for each individual input setting $\boldsymbol{x}_{\boldsymbol{D} \boldsymbol{i}}$, and $i=1$ to $m$, where $m$ is the number of settings for which the outputs are observed. Note that the likelihood function is based on the joint PDF of the observations $\boldsymbol{y}_{\boldsymbol{D} i}$, conditioned on $\boldsymbol{\theta}, \boldsymbol{\sigma}_{\boldsymbol{o b s}}$ and $\boldsymbol{\varepsilon}_{\boldsymbol{d}}$.

Considering the likelihood function in this manner allows the inclusion of correlated model outputs, correlated model errors, and correlated sensor observations. The formulation in Eq. (6) is general, and can handle correlated as well as independent parameters. In the case where the observed outputs are independent, the likelihood in Eq. (6) is the product of the likelihoods for the individual outputs as well as samples $y_{D i j}(i=1$ to $m, j=1$ to $n)$, as:

$$
L\left(\boldsymbol{\theta}, \boldsymbol{\sigma}_{\boldsymbol{o b s}}, \boldsymbol{\varepsilon}_{\boldsymbol{d}}\right) \propto \prod_{i=1}^{m} \prod_{j=1}^{n} \pi\left(y_{D i j} \mid \boldsymbol{x}_{D i}, \boldsymbol{\theta}, \sigma_{o b s j}, \varepsilon_{d j}\right)
$$


The model discrepancy term $\boldsymbol{\varepsilon}_{\boldsymbol{d}}$ has contribution from many sources of modeling error (numerical as well as model form) [11] [12]. In this paper, we do not quantify the individual model error sources, but only the overall discrepancy term because the focus is on the calibration of system parameters.

In order to get accurate calibration results, both modeling and experimental errors need to be included in the calibration [32]. Research has been done on identifying the characteristics of the prediction error, using available experimental observations. Christodoulou and Papadimitriou [55] and Zhang et al. [56] include variance parameters of uncorrelated zero-mean Gaussian models of experimental measurement errors, and correlation parameters like correlation lengths [57, 58]. The experimental measurement errors $\boldsymbol{\varepsilon}_{\boldsymbol{i n}}$ (input measurement error) and $\boldsymbol{\varepsilon}_{\boldsymbol{o b s}}$ (output measurement error) are represented here as random variables, with known (or assumed) distributions. In the numerical example, the scarcity of experimental data available does not allow further characterization of these errors.

Two issues need to be addressed in quantifying the model discrepancy term - capturing of missing physics and assumption of prior distributions for the discrepancy term parameters. Brynjarsdottir and O'Hagan [34] showed that satisfying calibration results can only be attained if the discrepancy captures the missing physics in the model; thus, there is need for a rigorous discrepancy model. This requires the inclusion of additional parameters in the discrepancy term, then additional parameters for calibration. This may slow down the convergence in the best case scenario, increase the uncertainty of calibration results, and sometimes even lead to parameter non-identifiability [58]. Many prior assumptions for the model discrepancy term $\varepsilon_{d}$ have been used in the literature, including constant, random walk [59], physics-based deterministic function [60], Gaussian random variable [61], uncorrelated random vector [62], and Gaussian random 
process [63]. It is calibrated along with model parameters using experimental data, following Eq. (5). Typically, well-designed high fidelity models present a smaller $\boldsymbol{\varepsilon}_{\boldsymbol{d}}$ than lower fidelity models. However, high-fidelity models may be prohibitively expensive in some problems.

The surrogate model error reflects the uncertainty we have regarding the replacement of the original model with a response surface model, as is shown in Eq. (4), and is affected by the number of training points available from the model simulations. Since lower fidelity models are usually much cheaper to run than higher fidelity ones, low fidelity simulations are generally more numerous, and result in a reduced surrogate error. To illustrate this, let $N_{l}$ and $N_{2}$ denote the number of simulations available for each original model $G_{l}(\boldsymbol{X}, \boldsymbol{\theta})$ and $G_{2}(\boldsymbol{X}, \boldsymbol{\theta})$, uniformly distributed over the problem domain $\left(G_{l}(\boldsymbol{X}, \boldsymbol{\theta})\right.$ and $G_{2}(\boldsymbol{X}, \boldsymbol{\theta})$ refer to lower and higher fidelity models respectively). These realizations are used as training points to build the corresponding surrogate models. Because of time and budget constraints, we assume that the higher the fidelity of the model, the lower the number of simulations available, i.e.: $N_{2}<N_{l}$. This results in a surrogate model error for $G_{2}(\boldsymbol{X}, \boldsymbol{\theta})$ larger than that for $G_{1}(\boldsymbol{X}, \boldsymbol{\theta})$. However, since $G_{2}(\boldsymbol{X}, \boldsymbol{\theta})$ is of a higher fidelity than $G_{l}(\boldsymbol{X}, \boldsymbol{\theta})$, the model discrepancy term in $G_{l}(\boldsymbol{X}, \boldsymbol{\theta})$ is larger than that for $G_{2}(\boldsymbol{X}$, $\boldsymbol{\theta}$ ). Fig. 4 shows a notional diagram of how the surrogate model error and the model discrepancy term might vary with the fidelity of the model.

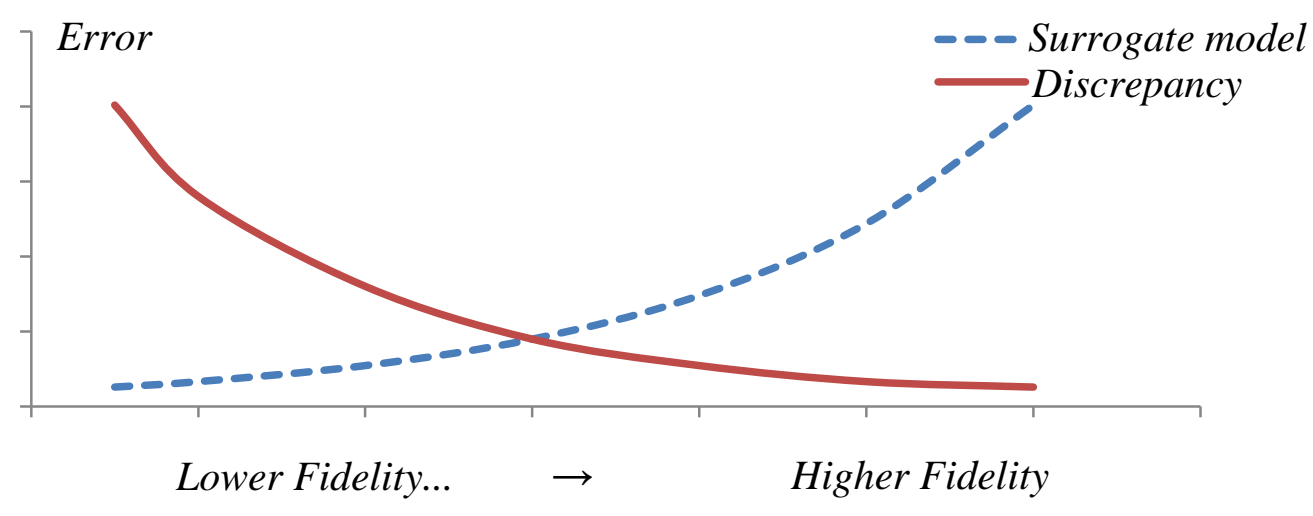

Fig. 4: Variation of surrogate model error and discrepancy with model fidelity 


\subsection{Multi-Fidelity Calibration Method}

The proposed approach avoids building a surrogate model for the high-fidelity simulations, and thus avoids the high surrogate model error that comes with it. It uses the available simulations of the high-fidelity model only to improve the low-fidelity surrogate model, and uses the improved low-fidelity model in the calibration process. Two options are possible: The first is a bias correction method, that consists of calibrating the discrepancy between the high and low fidelity models only, and the second consists of calibrating both the LF model parameters $\left(\theta_{i}\right)$ and the discrepancy term between the models $\left(\boldsymbol{D}_{2,1}\right)$ using HF simulations.

\subsubsection{The Bias Correction Method}

One option for improving the low fidelity model is by bias correction alone of the low fidelity model, i.e., simply calculating the difference between the low and high fidelity model outputs for the same input setting and adding that difference to the low fidelity model during the parameter calibration.

A serious challenge for this approach is the low number of high fidelity simulations available, because of the computational cost. In that case, the low fidelity model would be run at the high fidelity simulation's input values, and the difference between these outputs will be used to build a surrogate model for the correction term $\boldsymbol{D}_{2,1}$, in order to calculate the correction term at input conditions not considered in the high fidelity simulations. As a result, in the same manner as discussed in section 3.1 with respect to the surrogate of the high fidelity model, the surrogate model for the correction term will also incur a high error due to the low number of training points (limited by the number of high fidelity simulations). 


\subsubsection{The Pre-Calibration Method}

In the pre-calibration method, the HF results are used to update the distributions of the parameters in the LF model, and then the updated distributions are used as priors in the second step of calibration with actual experimental data. The assumption is that the HF model retains physics that might not be present in the LF model, and that they should influence the values of the parameters as well as the discrepancy, thus providing stronger physics-informed priors for calibration. This is an improvement of the bias-correction method, where the missing physics from the LF model that are present in the HF model are lumped into a single correction term, which has significant error due to the low number of training points (see Section 3.2.1).

The proposed multi-fidelity pre-calibration algorithm is as follows:

i. Run the Low $\left(G_{1}\left(X, \theta_{i}\right)\right)$ and High $\left(G_{2}\left(X, \theta_{i}\right)\right)$ fidelity models to obtain $N_{1}$ and $N_{2}$ sets of outputs, respectively.

ii. Build $S_{l}\left(\boldsymbol{X}, \theta_{i}\right)$, the surrogate model replacing $G_{l}\left(\boldsymbol{X}, \theta_{i}\right)$. In this step, the variance $\boldsymbol{\varepsilon}_{\text {surr }}$ of $S_{l}(\boldsymbol{X}$, $\left.\theta_{i}\right)$ is also calculated to account for the surrogate model prediction uncertainty.

iii. Define the priors of the calibration parameters $\theta_{i}$, and the discrepancy between the models $D_{2,1}$.

iv. Update the parameters of low-fidelity model as well as the discrepancy term with the highfidelity simulation results, i.e., use the relationship

$$
\boldsymbol{Y}_{\boldsymbol{H F}}=S_{l}\left(\boldsymbol{X}, \theta_{i}\right)+\boldsymbol{\varepsilon}_{\text {surr }}+\boldsymbol{D}_{2,1}
$$

to compute the posterior distributions of $\theta_{i}$ and $\boldsymbol{D}_{2,1}$, denoted as $\theta_{i}$ 'and $\boldsymbol{D}_{2,1}$ respectively.

v. Define the corrected low-fidelity surrogate model with the updated parameters $\theta_{i}$ 'and the updated discrepancy $\boldsymbol{D}_{2,1}$ as

$$
\boldsymbol{L F}_{\text {corr }}=S_{1}\left(\boldsymbol{X}, \theta_{i}{ }^{\prime}\right)+\boldsymbol{D}^{{ }_{2,1}}
$$


vi. Assume a prior distribution for the model discrepancy term $\boldsymbol{\varepsilon}_{\boldsymbol{d}}$ (i.e., the difference between model prediction and experimental observation).

vii. Re-calibrate the dynamics model parameters along with $\varepsilon_{d}$ with the available experimental data, using the relationship

$$
\boldsymbol{Y}_{\text {obs }}=S_{1}\left(\boldsymbol{X}, \theta^{\prime}{ }_{i}\right)+\varepsilon_{\text {surr }}+\boldsymbol{D}^{\boldsymbol{\prime}_{2,1}}+\boldsymbol{\varepsilon}_{\boldsymbol{d}}
$$

using the posteriors of the dynamics model parameters from step iv. as priors, to compute updated parameters $\theta_{i}{ }^{\prime}$ and the updated model discrepancy $\boldsymbol{\varepsilon}_{\boldsymbol{d}}$. (Note that $\boldsymbol{\varepsilon}_{\text {surr }}$ and $\boldsymbol{D}_{2,1}{ }_{2,1}$ are fixed in this step based on the results of step ii and step $v$ respectively).

The multi-fidelity calibration approach is particularly useful when models of varied fidelities retain different physical properties of the system. When calibrating the low fidelity model using high fidelity simulations, the parameters to which the high fidelity model are most sensitive see a substantial shift in their distributions. The more sensitive the high fidelity model is to the parameters, the less uncertainty the parameter distributions will have in step $v$. Using these distributions as priors in step vii ensures the retention of high fidelity information with respect to the parameter values before calibration with experimental data. This also reduces the computational cost by effectively shrinking the parameter samplig space in step vii. The parameters are only sampled in the posterior region (defined in step $v$ ), not in the full range covered by the original priors.

Although high fidelity models may be more exact, the lower fidelity ones usually capture the global behaviour of the system, mainly because the high fidelity simulation cannot be run over the full domain because of limited resources. In dynamical systems, fusion of information from multiple fidelities is valuable because lower fidelity models (e.g., PSD analysis), retain the average energy response, whereas higher fidelity models, (e.g., time history analysis), preserves 
the peaks in the signals (two very different signals can have the same PSD graph, if their energy peaks at the same frequencies). Also, a PSD analysis output is only derived from forces varying over time. This means initial conditions on the structure are not included in the calculations, which can influence the final result. A full time history analysis is not limited by this condition, and any constant force on the system affects the output. Moreover, typically, PSD analyses combine modal static forces, which can be translated into (stiffness) times (mode shape forces). In a full solution method (time history), inertia, damping and static loads are used to calculate the nodal reaction loads at each time step. This has a direct influence on how stiff the model is, and how the damping effect is expressed. The pre-calibration method allows the transfer of the physics from the HF model to the LF model through the paramters as well as the dispcrepency term because of stronger, physics-informed priors of these parameters. These differences influence the sensitivety of these models to various input parameters, and thus the accuracy of the calibration results.

In order to assess the proposed apporach, the multi-fidelity calibration results are compared to calibrations done with the single fidelity models (low and high), using Bayesian hypothesis testing, as discussed in section 3.3.

\subsection{Model Comparison}

Model comparison is done using experimental data (independent of the data used in the calibration process) and checking how much it supports one model versus the other. Several comparison techniques exist, ranging from graphical visual comparison, to different types of hypothesis testing. Berger and Pericchi [64] argue that Bayesian methods of model selection and hypothesis testing are needed because measures based on frequentist computations are 
ambiguous and do not allow for comparing multiple models. Another notable difference between Bayesian and classical hypothesis testing is that the first picks the model that is most likely to be accepted, whereas the second rejects the model that does not have enough evidence supporting it [65].

Consider two models $\left(M_{1}\right.$ and $\left.M_{2}\right)$, with outputs $Y_{1}$ and $Y_{2}$, and prior probabilities of acceptance $P\left(Y_{1}\right)$ and $P\left(Y_{2}\right)$. The output PDFs are denoted $f_{\boldsymbol{X}}^{1}(\boldsymbol{x}, \boldsymbol{\Theta})$ and $f_{\boldsymbol{X}}^{2}(\boldsymbol{x}, \boldsymbol{\Theta})$ respectively, where $\boldsymbol{\Theta}$ includes all the calibration quantities in Eq. (6) ( $\boldsymbol{\theta}, \boldsymbol{\sigma}_{\boldsymbol{o b s}}$ and $\left.\boldsymbol{\varepsilon}_{\boldsymbol{d}}\right)$. The relative posterior probabilities based on observed data can be computed using Bayes' rule [66]:

$$
\frac{P\left(\boldsymbol{Y}_{1} \mid \boldsymbol{Y}_{o b s}\right)}{P\left(\boldsymbol{Y}_{2} \mid \boldsymbol{Y}_{o b s}\right)}=\frac{P\left(\boldsymbol{Y}_{o b s} \mid \boldsymbol{Y}_{1}\right)}{P\left(\boldsymbol{Y}_{o b s} \mid \boldsymbol{Y}_{2}\right)} \frac{P\left(\boldsymbol{Y}_{1}\right)}{P\left(\boldsymbol{Y}_{2}\right)}
$$

The likelihood ratio $B=\frac{P\left(\boldsymbol{Y}_{\text {obs }} \mid \boldsymbol{Y}_{1}\right)}{P\left(\boldsymbol{Y}_{\text {obs }} \mid \boldsymbol{Y}_{2}\right)}$ is referred to as "Bayes factor" [67], and is used as the metric to assess the data support to model $M_{1}$ relative to model $M_{2}$.

If the Bayes factor $B$ is greater than 1.0, then it can be concluded that the model $M_{1}$ has greater support from the data. The higher the value of $B$, the greater the confidence is in picking $M_{1}$ over $M_{2}$. If the prior probabilities of the two model predictions $\mathrm{P}\left(\boldsymbol{Y}_{1}\right)$ and $\mathrm{P}\left(\boldsymbol{Y}_{2}\right)$ are equal, then in fact we can derive

$$
\begin{aligned}
& P\left(\boldsymbol{Y}_{\boldsymbol{I}} \mid \boldsymbol{Y}_{o b s}\right)=\frac{B}{B+1}, \text { and } \\
& P\left(\boldsymbol{Y}_{2} \mid \boldsymbol{Y}_{o b s}\right)=\frac{1}{B+1}
\end{aligned}
$$

This gives a convenient quantitative measure of the confidence in selecting model $M_{1} \mathrm{vs}$. $M_{2}$. 
In order to implement the above Bayesian hypothesis testing, the likelihood functions $P\left(Y_{o b s} \mid Y_{1}\right)$ and $P\left(Y_{o b s} \mid Y_{2}\right)$ need to be calculated. This is done in two steps. Assuming $m$ data points available, we first calculate $P\left(Y_{o b s} \mid Y_{1}, \Theta\right)$

$$
P\left(\boldsymbol{Y}_{\boldsymbol{o b s}} \mid \boldsymbol{Y}_{\boldsymbol{1}}, \boldsymbol{\Theta}\right) \propto L\left(\boldsymbol{Y}_{\boldsymbol{1}}, \boldsymbol{\Theta}\right)=\prod_{i=1}^{m} f_{X}^{1}\left(\boldsymbol{x}=\boldsymbol{x}_{i} \mid \boldsymbol{\Theta}\right)
$$

then integrate it over $\theta$, to obtain

$$
L\left(\boldsymbol{Y}_{1}\right) \propto P\left(\boldsymbol{Y}_{\boldsymbol{o b s}} \mid \boldsymbol{Y}_{1}\right)=\int P\left(\boldsymbol{Y}_{\boldsymbol{o b s}} \mid \boldsymbol{Y}_{1}, \boldsymbol{\Theta}\right) f_{\boldsymbol{\Theta}}(\boldsymbol{\Theta}) d \boldsymbol{\Theta}
$$

where $f_{\boldsymbol{\Theta}}(\boldsymbol{\Theta})$ denotes the posterior PDF of $\boldsymbol{\Theta}$ after calibration.

The same equations can be written for $M_{2}$ and $Y_{2}$, and $P\left(Y_{o b s} \mid Y_{2}, \Theta\right)$ can be calculated.

In this paper, the following three calibration approaches (treated as models) are compared:

- $\quad$ The low fidelity model calibrated using experimental data (LF)

- The high fidelity model calibrated using experimental data (HF)

- The low fidelity model pre-calibrated with HF simulations then re-calibrated with the experimental data (LFcorr).

In order to compare the three models using the Bayes factor, we calculate the likelihood of observing the experimental data (independent of the data used in the calibration process) for each model, then normalize them with respect to the likelihood of observing the data in the LF model. The results are displayed as

$$
1: \frac{L_{H F}}{L_{L F}}: \frac{L_{L F c o r r}}{L_{L F}}
$$

where $L$ represents the likelihood, and the subscript denotes the model used. 


\section{NUMERICAL EXAMPLE}

\subsection{Problem Description}

The example problem is of a hypersonic airplane fuselage panel located next to the engine, subjected to dynamic acoustic loading (AL). The panel is curved, as shown in Fig. 5, and is modeled using the FEA software ANSYS. The objective is to calibrate the damping coefficient of the panel, using experimental strain data observed under applied acoustic loading. The strain is recorded at seven different locations of the panel. Three strain gages are placed on the top (SG1, SG2 and SG3), two on the bottom (SG4 and SG5) as shown in Fig. 5, with the center gage recording strain in three different directions. The experiments are performed in room temperature, at varying levels (decibels) of acoustic excitation. In this paper, the damping is calibrated under one level of excitation only $140 \mathrm{~dB}$ for the sake of illustration.

No repeated measurements under the same input are available. This hinders the ability to calculate any spatial correlation between the strain gages. However, repeated runs of the high fidelity simulations allow the calculation of the spatial correlation between the outputs at those locations. The correlation coefficient was found to be equal to 0.85 among the model outputs at the three strain gage locations, and we used the same correlation coefficient among the observations, observation errors, and model discrepancies at the three locations.

The experimental strain measurement is saved for a duration of sixty seconds, at a frequency of 50,000 recordings per second, for a total of three million data points. In order to compare the experimental signal to the simulation outputs, the strain signal in the time domain is transformed into a power spectral density (PSD) in the frequency domain, and the energy under the PSD curve, i.e., the root mean square (RMS) value of the signal (the area under the curve) is calculated. 
The PSD describes how the power of a signal or time series is distributed over different frequencies. For a signal $X(t)$, it is calculated as follows:

$$
S_{x x}(\omega)=\lim _{T \rightarrow \infty}\left[\frac{E\left[\left|F_{X_{T}}(\omega)\right|^{2}\right]}{2 T}\right]
$$

where $E[]$ is the expected value, and $F_{X_{T}}(\omega)=\int_{-\infty}^{\infty} X_{T}(t) e^{-j w t} d t$ the Fourier transform of $X(t)$.

A microphone, located near the center of the panel, captures the acoustic load seen by the panel as it vibrates. This measurement is used as the input load on the simulated structure.
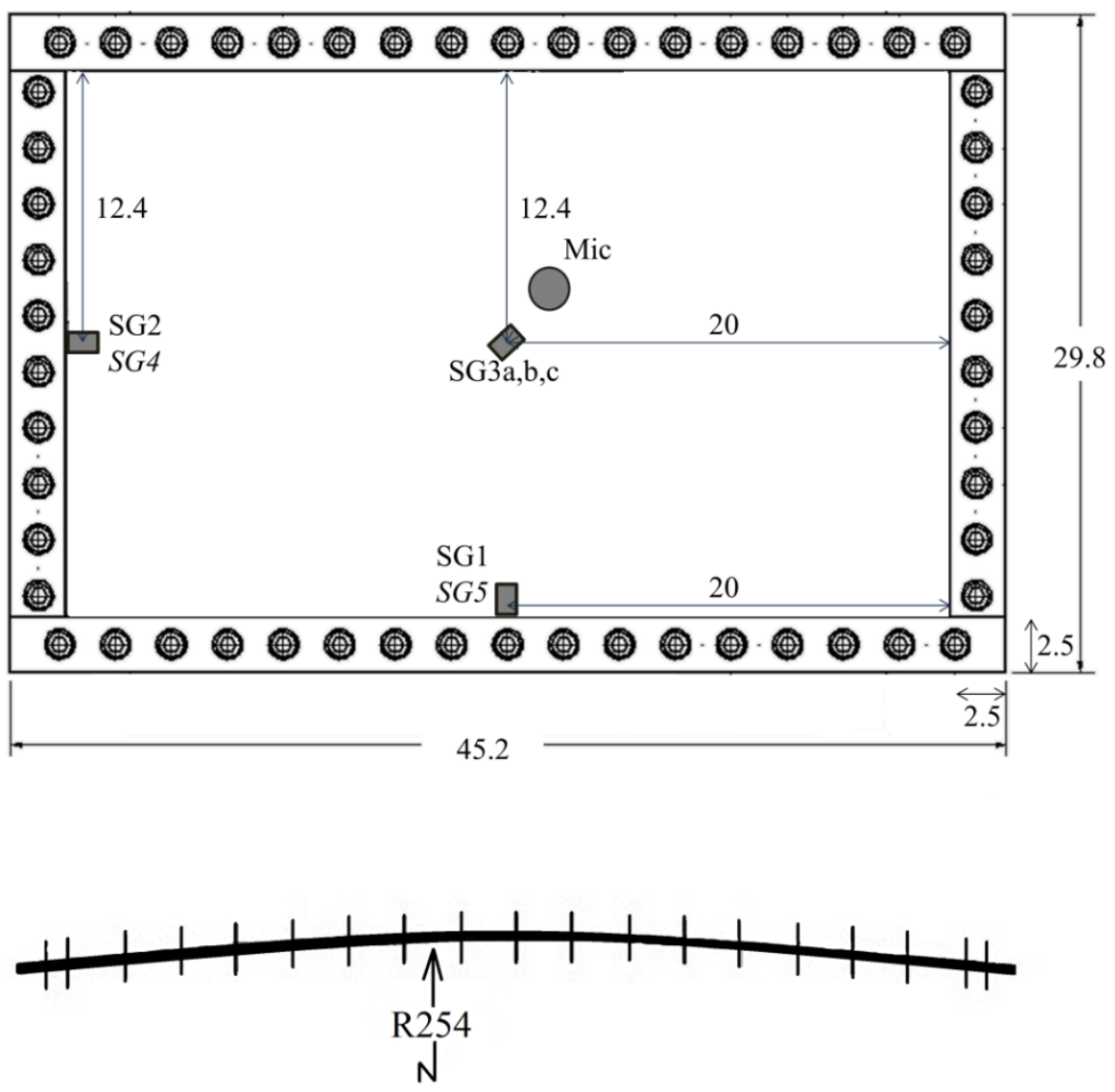

Fig. 5: Curved panel dimensions and strain gage locations (units: $\mathrm{cm}$ ) 
The material damping, in this study, is modeled as a viscous damping throughout the panel. In addition, and due to the panel attachment boundaries (the edge of the plate is sandwiched between two 1" wide metal strips, and bolted to the test frame, as shown in Fig. 5), when the acoustic load is applied on the plate, a constant mechanical damping is expressed in which energy is absorbed via sliding friction. This form of damping is defined as Coulomb damping [68], and we denote it as frictional damping for a width of 1" around the perimeter of the panel.

The boundary fixity is also a calibration variable, to account for the uncertainty in building the test setup (loose bolts, for ex.), and is described by a fixity ratio $F R=$ length of fixed plate boundary /total boundary length (see Fig. 6).
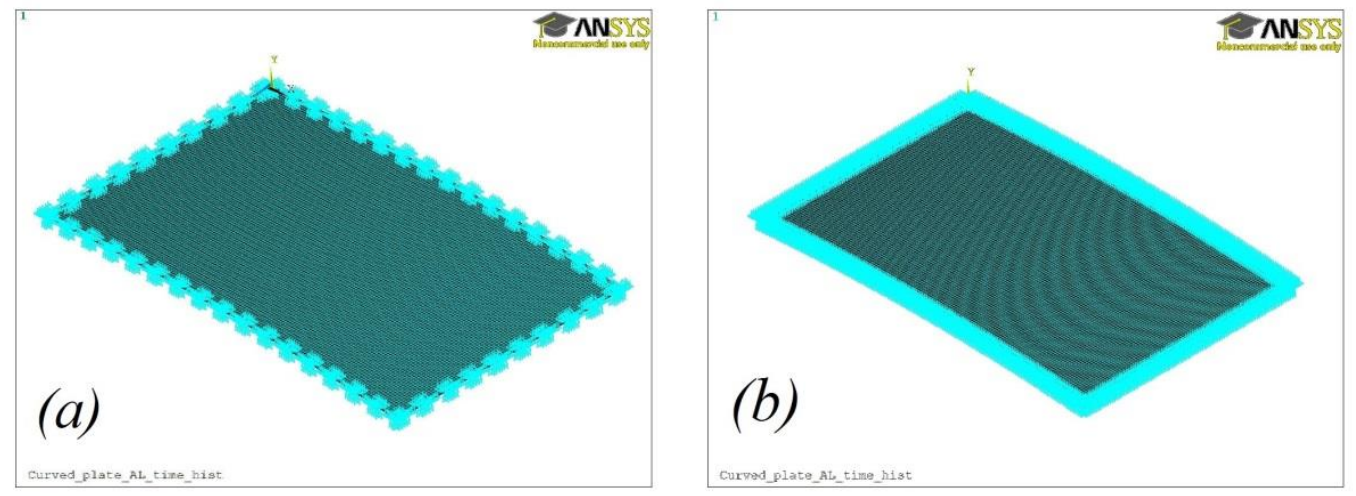

Fig. 6: Boundaries with different fixity ratios $(F R):(a) F R=0.5$, (b) $F R=1$

Two models of different fidelities were considered:

- $\quad$ Model 1: A power spectral density analysis, which consists of a linear combination of mode shape effects (referred to as low fidelity model).

- $\quad$ Model 2: A full transient analysis where the acoustic loading is applied as a dynamic time history input (referred to as high fidelity model). 
Model 1 and Model 2 have the same finite element mesh. In addition, the materials, the boundary conditions and the mesh resolution are similar. The models differ in the application of loads, the analysis method, and the output type.

In Model 1, the input acoustic load applied is the Welch power spectral density (PSD) [69] of the experimental $140 \mathrm{~dB}$ acoustic load. The PSD is calculated with the entire $140 \mathrm{~dB}$ signal, for the full duration of sixty sec. The output of Model 1 is a strain PSD curve. The strain RMS is calculated and used for comparison with the experimental data. This simulation is considered a lower-fidelity approximation of the experiment because the PSD input is not unique to the signal it is calculated from, since the phase component is discarded. For different phase relationships, different time-domain signals fit the same PSD. Also, the spectrum analysis in ANSYS is a linear combination of the mode effects on the structure. Finally, even though Model 1 is inexpensive to run (one simulation takes about 9.5 minutes to complete on a personal computer), it does not allow the user to add initial conditions on the structure. Initial stress resulting from fixing the plate on the test-rig (a uniform load has a PSD of zero) will not contribute to the RMS strain output.

Model 2, in contrast, is a full transient dynamic analysis of the panel. The output is a strain signal also converted to a PSD curve, and the strain RMS is derived from it. The model run is quite time-consuming, allowing only $0.2 \mathrm{sec}$ of the input signal (of the full $60 \mathrm{sec}$ of data available) to be simulated with the available computational resources, and each simulation takes 5.5 hours on a personal computer (35 times slower than Model 1). However, Model 2 does allow us to specify an initial stress on the panel, and preliminary test runs have shown that a uniform initial load on the panel is consistent with the experimental observation (See discussion in section 4.3 and Fig. 15). In Fig. 15, the initial stress specification is referred to as IC - Initial Conditions. 
The hypothesis of this paper is that initial improvement of the low-fidelity surrogate with a few runs of the higher fidelity model allows the use of information from both models in the final calibration of the system parameters. In this numerical example, the fused information consists of the full length of the signal in the form of the PSD, and the transient behavior as well as the initial conditions incorporated in the full dynamic analysis. The two models capture two different types of information, and both are effectively used in the proposed calibration strategy. This strategy also amounts to developing physics-informed stronger priors for calibration, thus reducing the uncertainty about the system parameters.

\subsection{Results}

Three calibration results are being compared in this section: (1) calibration of the low-fidelity surrogate model with experimental data (LF) using correlated parameters and uncertainties, (2) calibration of the high-fidelity surrogate model with experimental data (HF) using only correlated parameters (see next paragraph for explanation), and (3) calibration of the improved low-fidelity surrogate model with the experimental data (LFcorr) using correlated parameters and uncertainties. Table 1shows details of the surrogate model properties for each case.

Table 1: Surrogate model properties

\begin{tabular}{|l|l|l|l|}
\cline { 2 - 4 } \multicolumn{1}{c|}{} & LF & HF & LFcorr \\
\hline Surrogate type & PCE & PCE & PCE \\
Order & 2 & 1 & 2 \\
Training Points & 40 & 9 & 40 \\
\hline
\end{tabular}

The calibration parameters are: frictional damping $\left(F R \_D C\right)$, material damping $\left(M T \_D C\right)$, fixity ratio $(F R)$, as well as the discrepancy term $\left(\varepsilon_{d}\right)$ and standard deviation of the observation error $\left(\sigma_{o b s}\right)$. Since we are measuring three outputs, there are correspondingly three discrepancy terms 
and three observation errors. The priors for the calibration parameters are assumed the same for the LF, HF and LFcorr models. They are all uninformed priors (i.e. uniform distributions U(a,b)) for the model parameters, normal distributions $\left(\mathrm{N}\left(\boldsymbol{\mu}_{\boldsymbol{d}}, \boldsymbol{\sigma}_{\boldsymbol{d}}\right)\right)$ for the discrepancy terms with uniform priors for $\boldsymbol{\mu}_{\boldsymbol{d}}$ and $\boldsymbol{\sigma}_{\boldsymbol{d}}$, and Jeffrey's priors [70] for the $\boldsymbol{\sigma}_{\boldsymbol{o b s}}$ terms which is the correct choice of an uninformed prior for standard deviation, $\pi\left(\sigma_{o b s}\right) \propto 1 / \sigma$. The experimental error itself is assumed to follow a normal distribution with zero mean (Table 2). The same prior parameter distributions are assumed at all three strain gage locations, so they are not repeated in Table 2.

Table 2: Prior distributions of calibration parameters

\begin{tabular}{|l|l|l|l|}
\hline Calibration parameter & Dist. type & Parameter 1 & Parameter 2 \\
\hline$F R_{-} D C$ & Uniform & $\mathrm{a}=10^{-5}$ & $\mathrm{~b}=10^{-2}$ \\
$M T_{-} D C$ & Uniform & $\mathrm{a}=5.10^{-7}$ & $\mathrm{~b}=10^{-5}$ \\
$F R$ & Uniform & $\mathrm{a}=0.7$ & $\mathrm{~b}=1$ \\
\hline $\boldsymbol{\mu}_{\boldsymbol{d}}$ & Uniform & $\mathrm{a}=-10^{-2}$ & $\mathrm{~b}=10^{-2}$ \\
$\boldsymbol{\sigma}_{\boldsymbol{d}}$ & Uniform & $\mathrm{a}=5.10^{-8}$ & $\mathrm{~b}=5.10^{-6}$ \\
\hline $\boldsymbol{\sigma}_{\boldsymbol{o b s}}$ & Jeffreys' & $\mu=0$ & $\sigma=10^{-7}$ \\
\hline
\end{tabular}

Only one set of experimental data is available (one observation at each strain gage location). Three strain gage outputs are used for calibration (i.e., $n=3$ with respect to Eqs. (6) and (7)), and a fourth one is used to compute the Bayes factor (likelihood ratio) to compare the results of the different calibration options. The three remaining strain gages (out of the total seven placed on the plate) were discarded from this study because the values recorded were very low compared to the rest of the strain gages.

Fig. 7 shows the posteriors of one of the discrepancy terms (at strain gage location SG4) is shown for illustration purposes. The posteriors of frictional damping, material damping, and fixity ratio are shown in Figs. 8 to 10 . Note that the posterior densities are only meaningful 
within the ranges of the priors. The use of kernel density smoothing results in the posterior densities extending outside the priors in Figs. 8 to 10 (and also in Figs. 12 to 14).

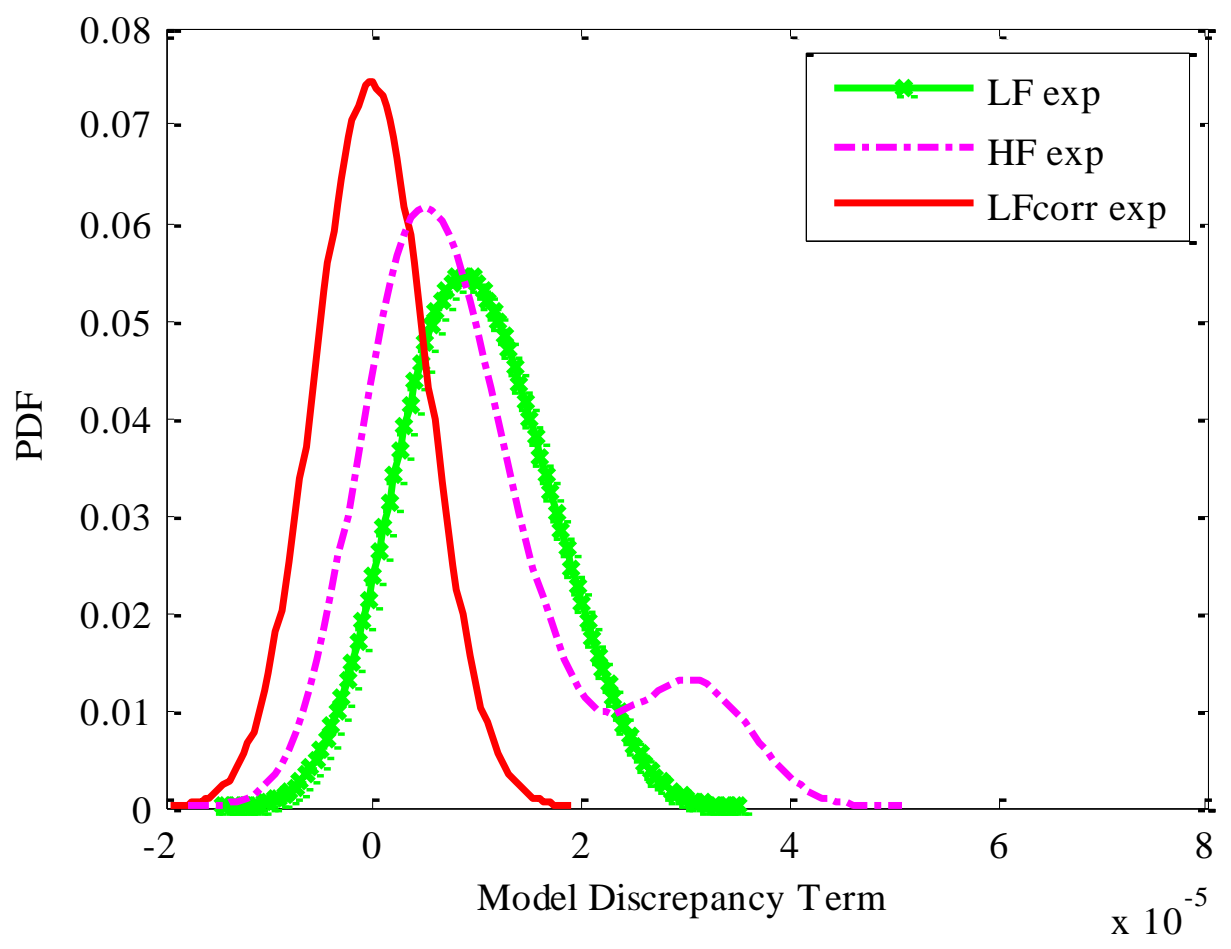

Fig. 7: Model discrepancy posteriors at strain gage location SG4 


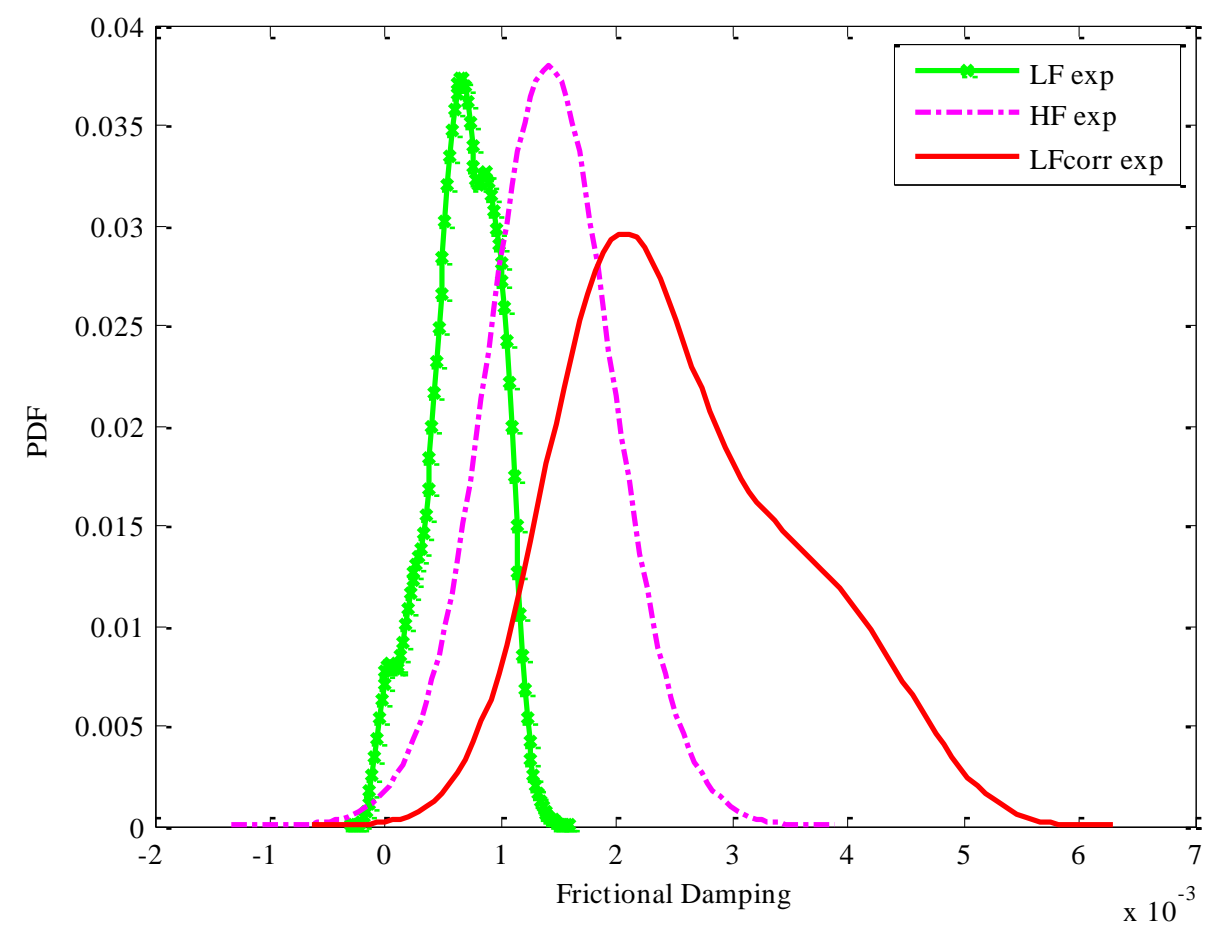

Fig. 8: Frictional damping posteriors. Prior Uniform [-10-5, 10-2]

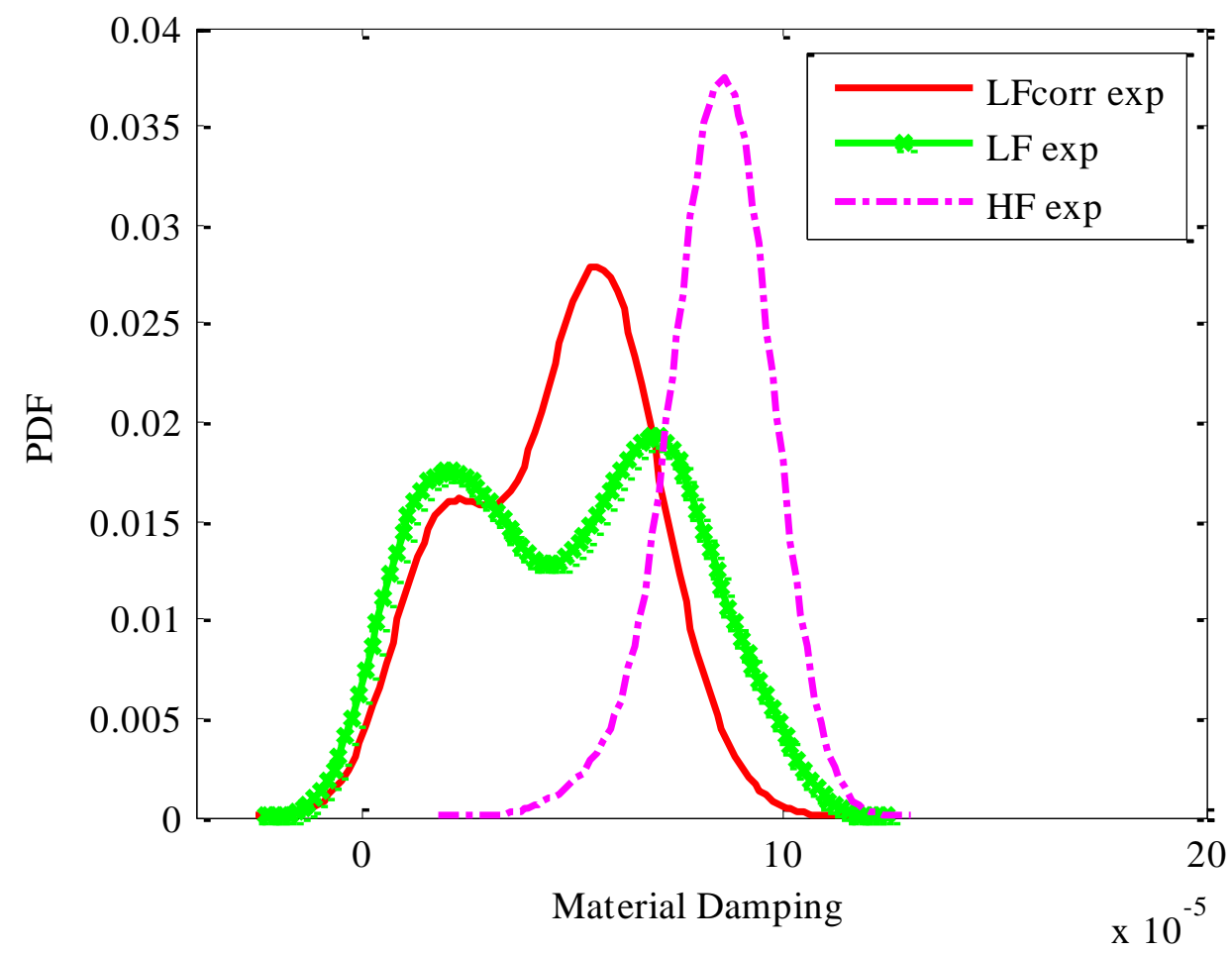

Fig. 9: Material damping posteriors. Prior Uniform [5.10-7, 10-2] 


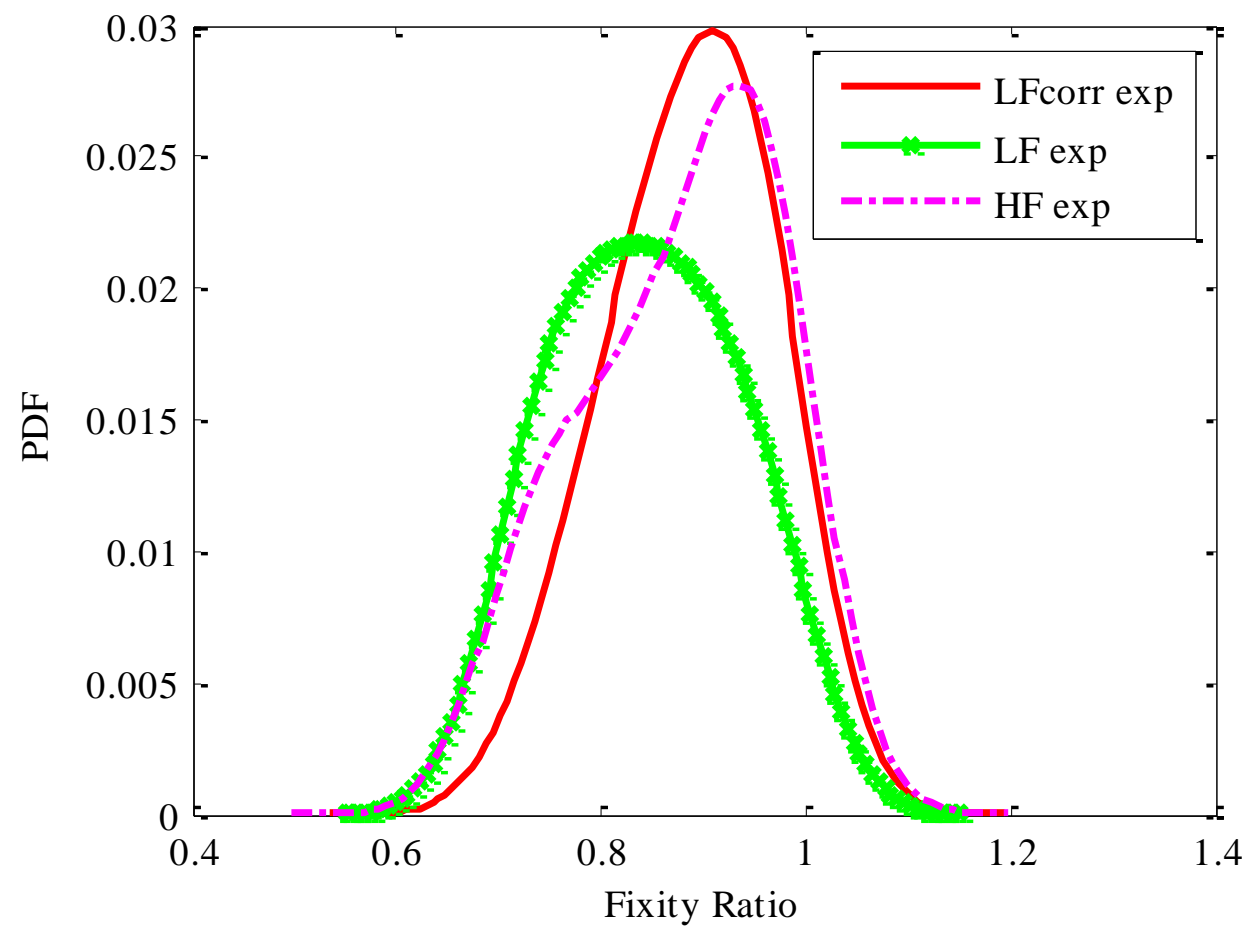

Fig. 10: Fixity ratio posteriors. Prior Uniform [0.7, 1]

The calibrations were also done for the case of independent model outputs and observations, and the associated errors, and Figs. 11 to 14 compare the posteriors of the LFcorr calibrations for the independent and correlated cases. 


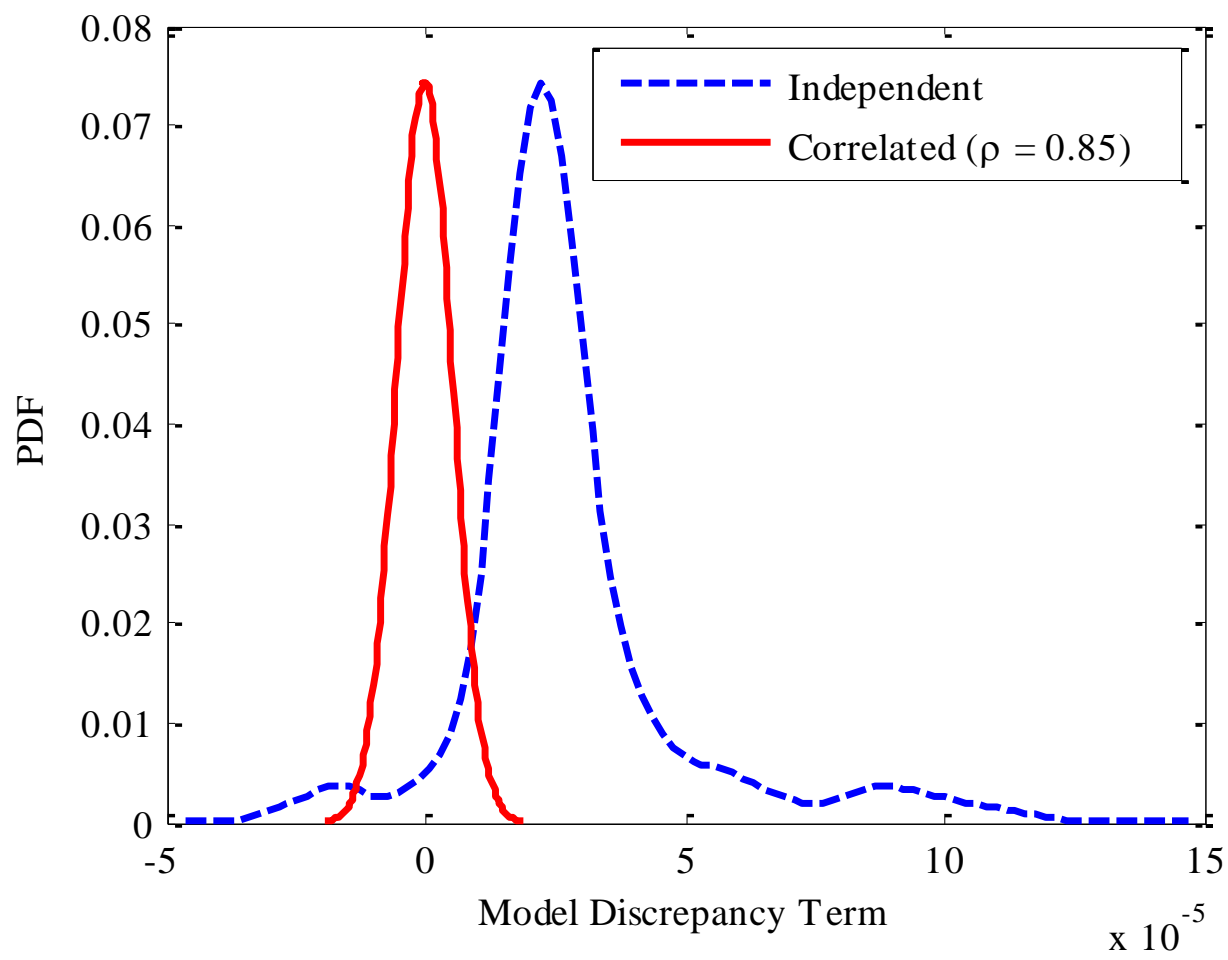

Fig. 11: Model discrepancy posteriors for independent and correlated parameters

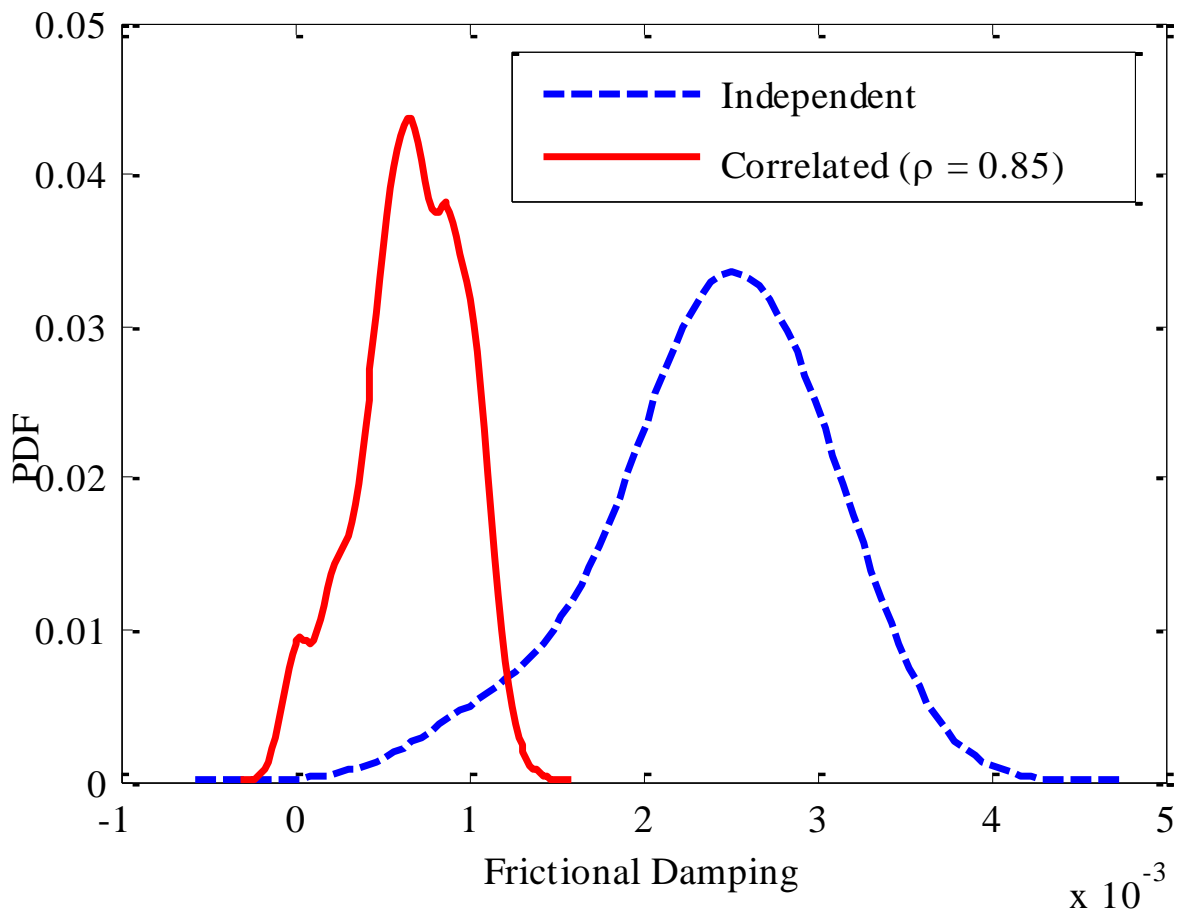

Fig. 12: Frictional damping posteriors for independent and correlated parameters 


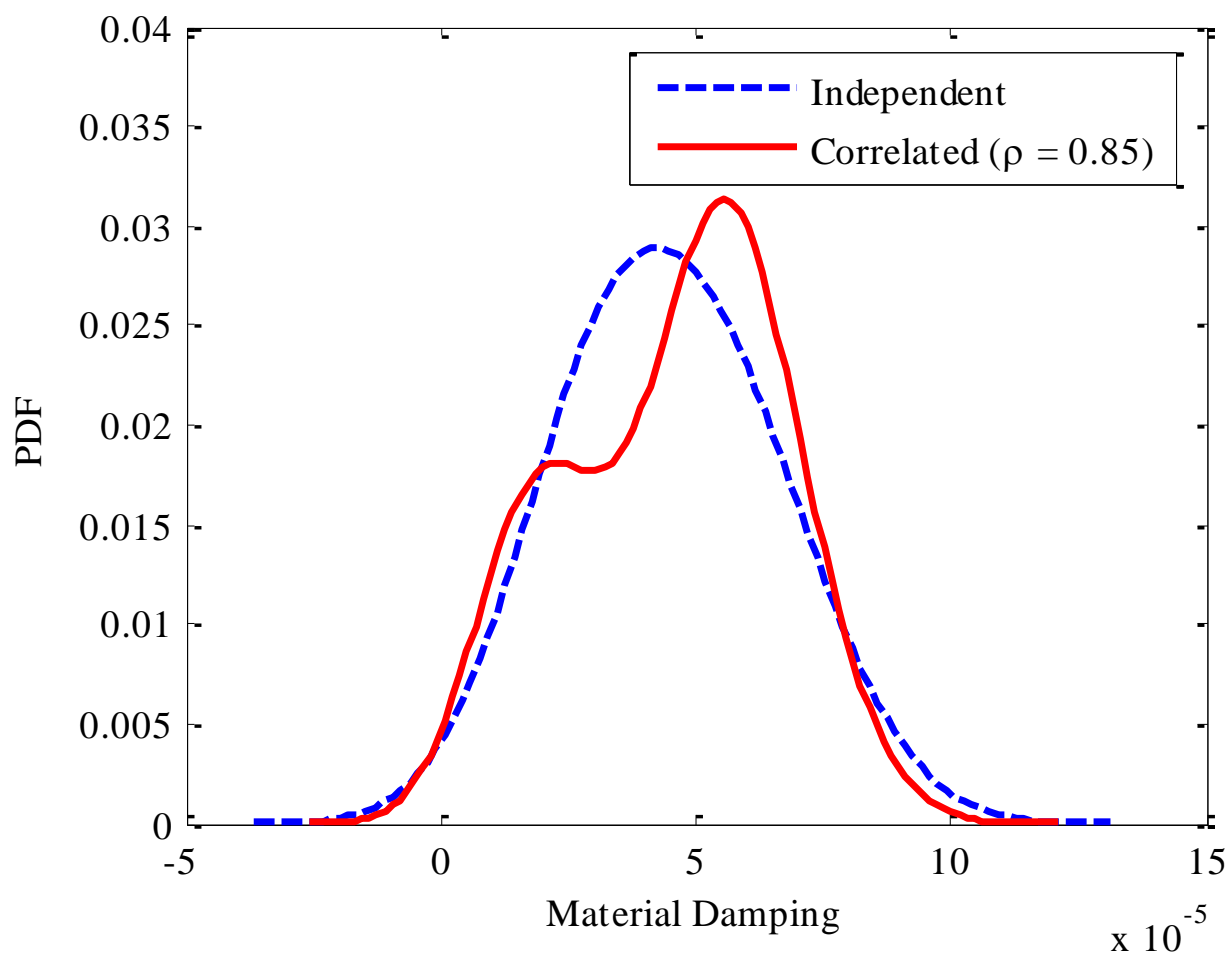

Fig. 13: Material damping posteriors for independent and correlated parameters

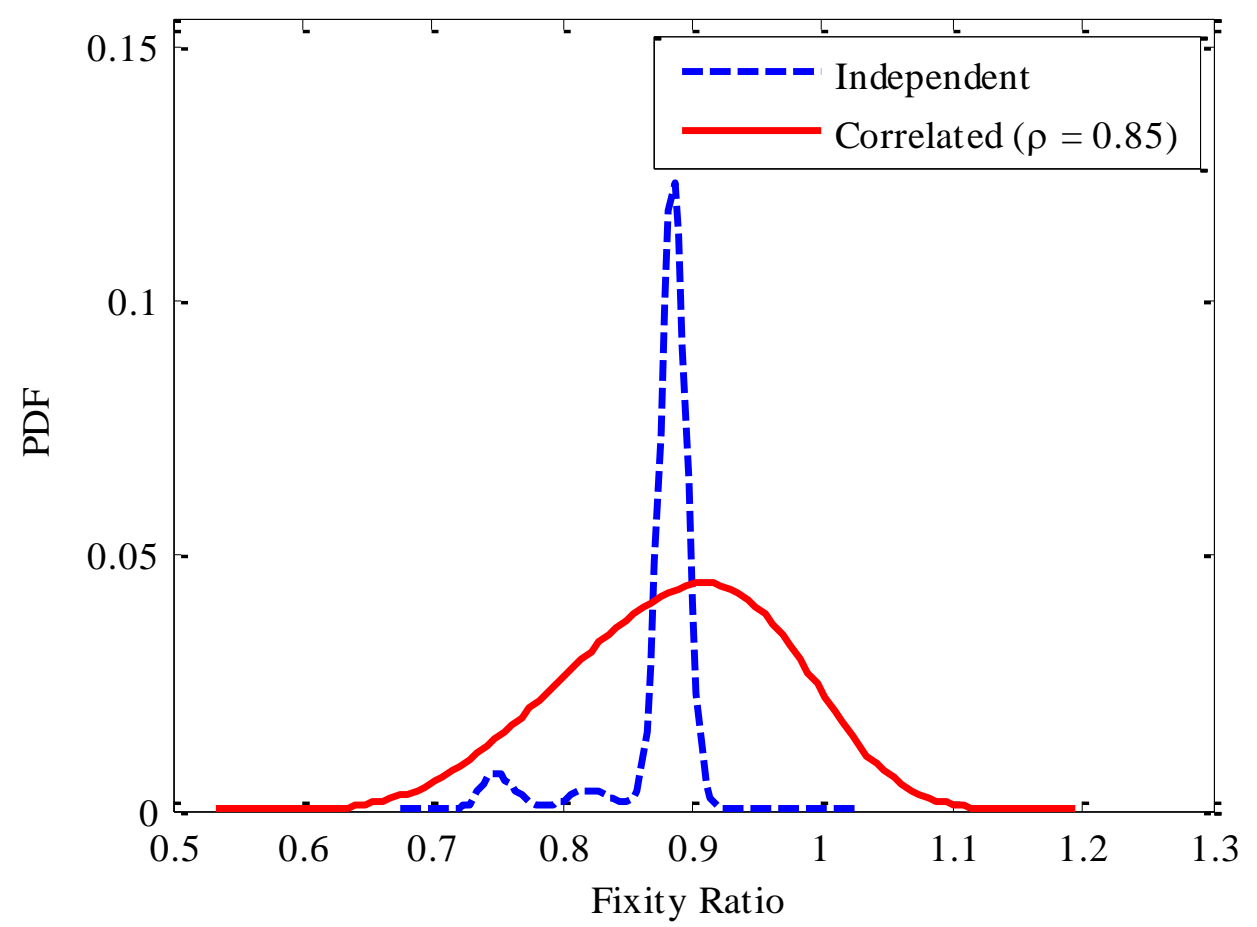

Fig. 14: Fixity ratio posteriors for independent and correlated parameters 
Using the observation at the fourth strain gage, the likelihood ratios among the four calibration options were computed as

$\mathrm{LF}: \mathrm{HF}: \mathrm{LFcorr}=1: 2.52: 3.97$

This indicates superior performance of the multi-fidelity calibration in predicting the response of the fourth strain gage compared to the individual low-fidelity and high-fidelity calibrations.

\subsection{Discussion}

The main difference between Models 1 and 2, besides the analysis type, is the initial stress added in Model 2, in the form of a distributed uniform load of $8.510^{-3} \mathrm{lb} / \mathrm{in}^{2}$. Preliminary testing of Model 2 with and without initial stress showed that, without adding a uniform load on the panel, the strain amplitude was much lower than the experimental output, as seen in Fig. 15. The initial stress is deforming the panel and allowing it to vibrate with a higher amplitude around a nonzero equilibrium.

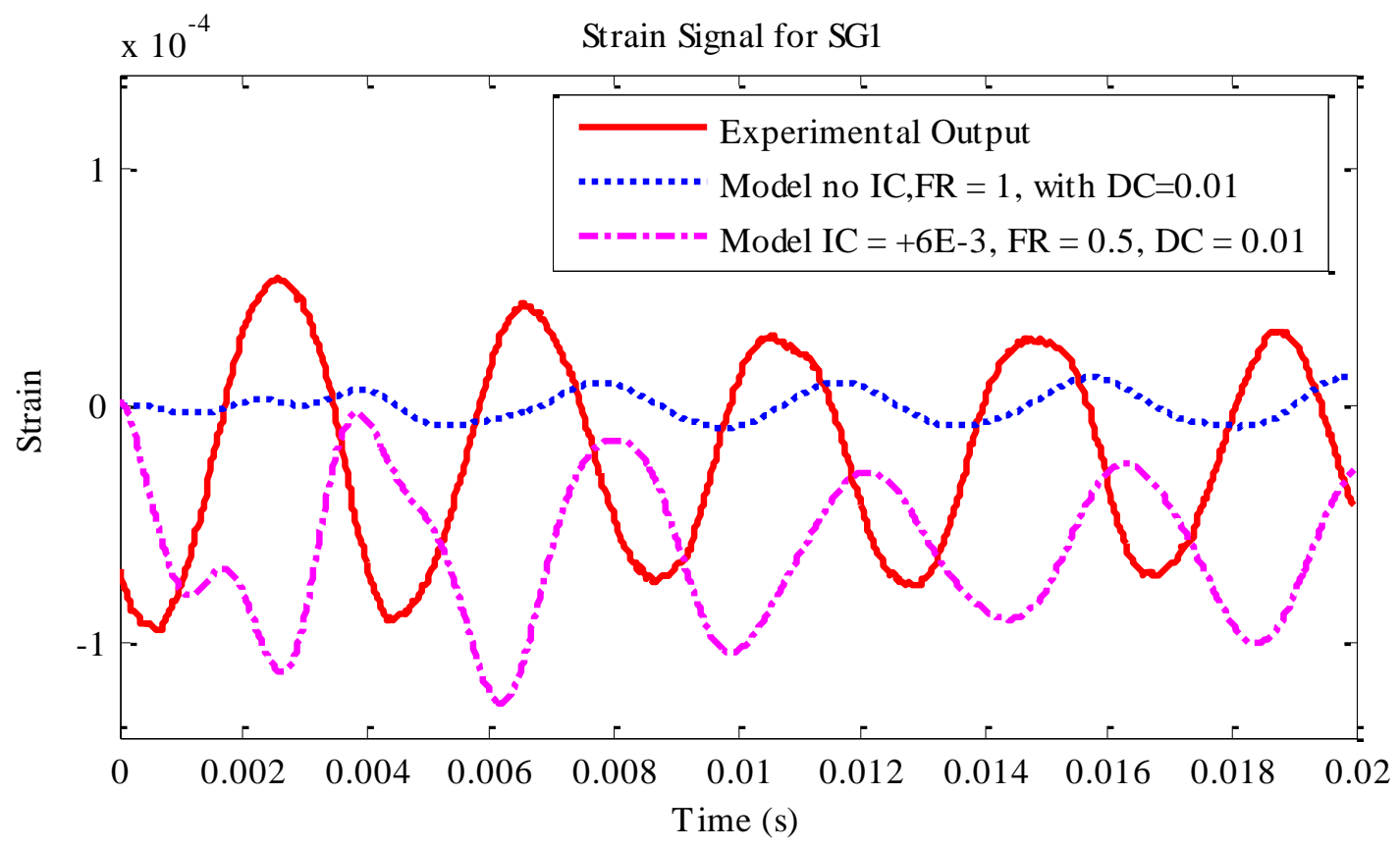

Fig. 15: Preliminary model testing 
The calibration results show the effect of ignoring the initial stress. The strain RMS is directly proportional to the signal amplitude, and in order to maximize the strain RMS from the low-fidelity model, the posteriors from the calibration with Model 1 underestimate the frictional damping and the fixity ratio, in an attempt to recover the energy under the PSD curve. This loss of accuracy is also shown in the discrepancy term, which is much greater (in magnitude) than the rest of the models.

It is observed that the LFcorr posteriors are closer to the low-fidelity posteriors for material damping, and are closer to the high-fidelity posteriors for frictional damping. Calibrating both model parameters and discrepancy of the low-fidelity model with high fidelity simulations allowed the retention of information from both models with respect to the parameters.

Most importantly, the results show the fusion of information from the low and high fidelity models in the proposed methodology, combining the different sensitivities of the HF and LF models to the parameters. A simple sensitivity analysis shows that, when the material damping is increased by $20 \%$, the strain PSD in SG1increases by $8 \%$ in the low-fidelity model (LF), and 5\% in the high-fidelity model (HF). On the other hand, a $20 \%$ increase in the frictional damping results in $2.5 \%$ increase of strain in LF, versus $15 \%$ in $\mathrm{HF}$. This shows that LF is more sensitive to material damping, whereas HF is more sensitive to frictional damping. Using the proposed calibration method, the final calibration results take advantage of the sensitivity of the low-fidelity and high fidelity models to different parameters. When using the corrected low fidelity model (LFcorr), the parameters' posteriors converge towards the posteriors of the models that are most sensitive to them, i.e., in the calibration of LFcorr, the material damping posterior converges towards the LF model posterior, and the frictional damping 
posterior converges towards the HF model posterior. This allows the fusion of information from both fidelities in a manner that captures the sensitivities of different fidelity models to different parameters.

Not only were the results in favor of the use of the improved low fidelity model in the calibration process, but its convergence was faster than both low fidelity and high fidelity calibrations with experimental data. This is due to multiple factors: the low fidelity model by itself has very low accuracy, but its surrogate has high precision, due to the high number of training points available. This makes convergence hard, since the low fidelity output is far from the experimental data. Conversely, the high fidelity model is more accurate, but its surrogate is considerably less precise due to the number of training points, and thus a high surrogate model error, which slows down the convergence considerably. The pre-calibration method results in two sequential smaller calibrations, where the first directs the second to the final converged answer. Effectively, the sampling space in the calibration with experimental data is reduced, since the priors used in this step consist of the posteriors coming from the calibration of the low fidelity surrogate with the high fidelity simulations. In other words, we are using stronger priors in the calibration with LFcorr, which causes faster convergence. The times to convergence (on a personal desktop computer) of all three analyses are shown in Fig. 16. 


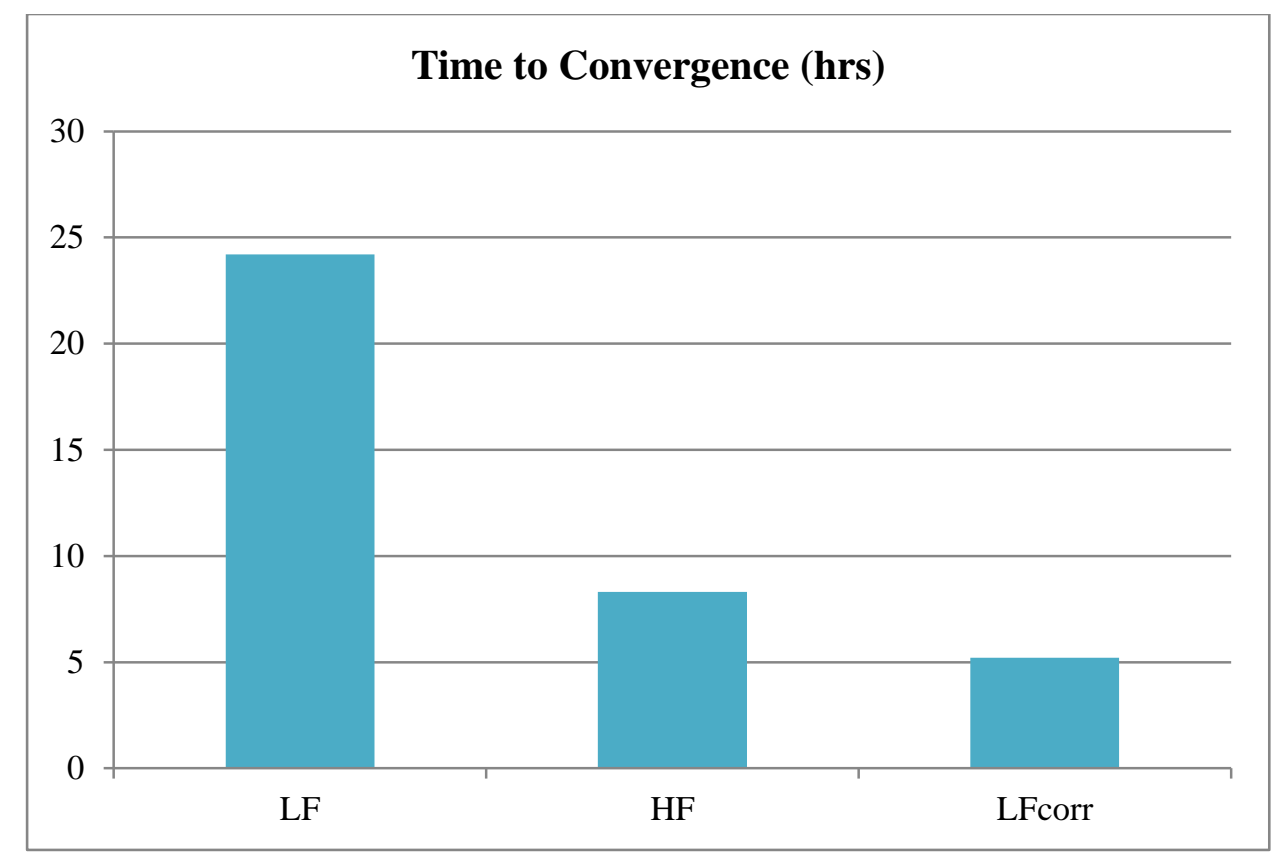

Fig. 16: Time to convergence of three calibration strategies

Furthermore, the likelihood ratio calculated in section 4.2 shows that the calibration result of the pre-calibrated low-fidelity model is much higher than all other models. When this likelihood comparison is considered along with the computational efficiency of the two-step approach, it appears that the proposed methodology offers a promising strategy in the use of multiple models of different fidelities in the calibration of model parameters.

As with any parameter estimation problem, the calibration results are only valid within the range of the test data. The formulation of the calibration procedure is general and is able to include correlations among model outputs, model errors as well as observation errors, as illustrated in the numerical example. The model discrepancy terms were represented in the numerical example either as unknown constants or normal random variables with unknown parameters. However, more elaborate representations of model discrepancy, such as inputdependent random field (following the Kennedy and O’Hagan approach) could also be included 
in the proposed formulation; such implementations are limited by the amount of data available. In the presence of limited data, the choice of priors also affects the calibration result.

\section{CONCLUSION}

This paper investigated a multi-fidelity approach for the Bayesian calibration of model parameters. Surrogate models become necessary in the context of Bayesian calibration due to the large number of model evaluations required in Markov Chain Monte Carlo sampling. A few time-consuming high-fidelity simulations were used to improve the surrogate of the inexpensive low fidelity model, and the improved low fidelity surrogate was used for parameter calibration with experimental data. This efficient two-step method allows the fusion of information from both model fidelities, and is particularly useful when only a small number of experimental data points are available, and calibration convergence is difficult with uninformed prior distributions of the parameters.

Future work needs to investigate the efficacy of this approach when more than two models are available (each with a different level of fidelity), and find a systematic quantitative way of using the available information, since multiple combinations are possible. The calibration becomes more complicated when the model parameter is input-dependent (e.g., load-dependent damping), and future work needs to account for this dependence. The extension of this approach to multi-physics problems also needs to be studied in the future, e.g., the application of combined acoustic, thermal and aerodynamic loads on the hypersonic panel considered in Section 4. 


\section{ACKNOWLEDGEMENT}

The research reported in this paper was partly supported by funds from the Air Force Office of Scientific Research (Project Manager: Dr. Fariba Fahroo) through subcontract to Vextec Corporation (Investigators: Dr. Robert Tryon, Dr. Animesh Dey). Valuable discussions with Dr. Joseph Hoelkampf at the Air Force Research Laboratory (Wright Patterson Air Force Base) are also acknowledged. The computations in the numerical example were partly conducted using the resources at the Advanced Computing Center for Research and Education (ACCRE) at Vanderbilt University. 


\section{REFERENCES}

[1] D. Zhou, Benefits of High-Fidelity Dynamic Simulation, ISA Automation Week, Mobile, Alabama, 2011.

[2] G.V. Candler, D.M. Peterson, T.W. Drayna, Detached Eddy Simulation of a Generic Scramjet Inlet and

Combustor, 47th AIAA Aerospace Sciences Meeting Including The New Horizons Forum and Aerospace

Exposition, Orlando, Florida, 2009.

[3] G.V. Candler, T. W., D. T.W., Design and Optimization of the ASET Inward-Turning Scramjet Inlet, JANNAF 30th Airbreathing Propulsion Subcommittee Meeting, Boston, Massachusetts, 2008.

[4] K. Higgins, S. Schmidt, Simulation of a Sonic Jet Injected into a Supersonic Cross-Flow, 16th Australasian Fluid Mechanics Conference, Gold Coast, Australia, 2007.

[5] M.W. Oppenheimer, T. Skujins, M.A. Bolender, D.B. Doman, A Flexible Hypersonic Vehicle Model Developed with Piston Theory, Atmospheric Flight Mechanics Conference and Exhibit, AIAA Paper No. 2007-6396, Hilton Head, South Carolina, 2007.

[6] M.A. Bolender, D.B. Doman, Nonlinear Longitudinal Dynamical Model of an Air-Breathing Hypersonic Vehicle, Journal of Spacecraft and Rockets, 44 (2007) 374-387.

[7] F.R. Chavez, D.K. Schmidt, Analytical aeropropulsive-aeroelastic hypersonic-vehicle model with dynamic analysis, Journal of Guidance, Control, and Dynamics, 17 (1994) 1308-1319.

[8] R. Oliva, Model calibration as a testing strategy for system dynamics models, European Journal of Operational Research, 151 (2003) 552-568.

[9] M.A. Christie, J. Glimm, J.W. Grove, D.M. Higdon, D.H. Sharp, M.M. Wood-Schultz, Error analysis and simulations of complex phenomena, Los Alamos Science, 29 (2005).

[10] S. Sankararaman, Y. Ling, S. Mahadevan, Uncertainty quantification and model validation of fatigue crack growth prediction, Engineering Fracture Mechanics, 78 (2011) 1487-1504.

[11] J.E. Mottershead, M.I. Friswell, Model Updating In Structural Dynamics: A Survey, Journal of Sound and Vibration, 167 (1993) 347-375.

[12] S. Mahadevan, B. Liang ERROR AND UNCERTAINTY QUANTIFICATION AND SENSITIVITY ANALYSIS IN MECHANICS COMPUTATIONAL MODELS, 1 (2011) 147-161.

[13] B. Caesar, Updating System Matrices Using Modal Test Data, 5th International Modal Analysis Conference, IMAC, London, England, 1987, pp. 453-459.

[14] M. McEwan, W. J.R., C. J.E., L. Y.T., A Finite Element / Modal Technique for Nonlinear Plate and Stiffened Panel Response Prediction, 42nd AIAA/ASME/ASCE/AHS/ASC Structures, Structural Dynamics, and Materials Conference ad Exhibit, AIAA-2001-1595, Seattle, Washington, 2001.

[15] M. McEwan, A Combined Modal / Finite Element Technique for the Non-Linear Dynamic Simulation of Aerospace Structures, University of Manchester, England, 2001.

[16] A.A. Muravyov, S.A. Rizzi, Determination of nonlinear stiffness with application to random vibration of geometrically nonlinear structures, Computers \& Structures, 81 (2003) 1513-1523.

[17] S.A. Rizzi, A. Przekop, System identification-guided basis selection for reduced-order nonlinear response analysis, Journal of Sound and Vibration, 315 (2008) 467-485.

[18] M. Mignolet, A. Radu, Validation of reduced order modeling for the prediction of the response and fatigue life of panels subjected to thermo-acoustic effects, Structural Dynamics: Recent Advances, 8th International Conference, University of Southampton, UK, 2003.

[19] K. Kim, X.Q. Wang, M.P. Mignolet, Nonlinear Reduced Order Modeling of Functionally Graded Plates, 49th AIAA/ASME/ASCE/AHS/ASC Structures, Structural Dynamics, and Materials Conference, AIAA-2008-1873, Schaumburg, Illinois, 2003.

[20] N.M. Alexandrov, R.M. Lewis, C.R. Gumbert, L.L. Green, P.A. Newman, Approximation and Model Management in Aerodynamic Optimization with Variable-Fidelity Models, Journal of Aircraft, 38 (2001) 1093 1101 .

[21] H. F., D. S., W. A., Discussion of Model Calibration and Validation for Transient Dynamic Simulation, 20th International Modal Analysis Conference, IMAC, Los Angeles, California, 2002, pp. 1362-1369.

[22] R.T. Haftka, Combining global and local approximations, AIAA Journal, 29 (1991) 1523-1525.

[23] M.G. Hutchison, E.R. Unger, W.H. Mason, B. Grossman, R.T. Haftka, Variable-complexity aerodynamic optimization of a high-speed civil transport wing, Journal of Aircraft, 31 (1994) 110-116.

[24] M. Kennedy, A. O'Hagan, Predicting the output from a complex computer code when fast approximations are available, Biometrika, 87 (2000) 1-13. 
[25] S. Leary, A. Bhaskar, A. Keane, A Knowledge-Based Approach To Response Surface Modelling in Multifidelity Optimization, Journal of Global Optimization, 26 (2003) 297-319.

[26] A.I.J. Forrester, N.W. Bressloff, A.J. Keane, Optimization using surrogate models and partially converged computational fluid dynamics simulations, 2006.

[27] M. Baruch, I.Y. Bar Itzhack, Optimal weighted orthogonalization of measured modes, AIAA Journal, 17 (1979) 927-928.

[28] A. Berman, E.J. Nagy, Improvement of a Large Analytical Model Using Test Data, AIAA Journal, 21 (1983) 1168-1173.

[29] M. Link, Updating of analytical models-Review of numerical procedures and application aspects, Structural Dynamics Forum SD2000, Los Alamos, New Mexico, 1999.

[30] B.N. Datta, S. Deng, V.O. Sokolov, D.R. Sarkissian, An optimization technique for damped model updating with measured data satisfying quadratic orthogonality constraint, Mechanical Systems and Signal Processing, 23 (2009) 1759-1772.

[31] Z.X. Yuan, K.P. Yu, Finite element model updating of damped structures using vibration test data under base excitation, Journal of Sound and Vibration, 340 (2015) 303-316.

[32] V. Arora, S.P. Singh, T.K. Kundra, Finite element model updating with damping identification, Journal of Sound and Vibration, 324 (2009) 1111-1123.

[33] M.C. Kennedy, A. O'Hagan, Bayesian calibration of computer models, Journal of the Royal Statistical Society: Series B (Statistical Methodology), 63 (2001) 425-464.

[34] J. Brynjarsdóttir, A. O’Hagan, Learning about physical parameters: the importance of model discrepancy, Inverse Problems, 30 (2014) 114007.

[35] E. Simoen, G. De Roeck, G. Lombaert, Dealing with uncertainty in model updating for damage assessment: A review, Mechanical Systems and Signal Processing, 56-57 (2015) 123-149.

[36] J.-A. Goulet, I.F.C. Smith, Structural identification with systematic errors and unknown uncertainty dependencies, Computers \& Structures, 128 (2013) 251-258.

[37] J.L. Beck, L.S. Katafygiotis, Updating Models and Their Uncertainties. I: Bayesian Statistical Framework, Journal of Engineering Mechanics, 124 (1998) 455-461.

[38] K.-V. Yuen, L.S. Katafygiotis, Bayesian Modal Updating using Complete Input and Incomplete Response Noisy Measurements, Journal of Engineering Mechanics, 128 (2002) 340-350.

[39] K.-V. Yuen, L. Katafygiotis, Bayesian Fast Fourier Transform Approach for Modal Updating Using Ambient Data, Advances in Structural Engineering, 6 (2003) 81-95.

[40] S.-K. Au, Fast Bayesian FFT Method for Ambient Modal Identification with Separated Modes, Journal of Engineering Mechanics, 137 (2011) 214-226.

[41] S.-K. Au, Fast Bayesian ambient modal identification in the frequency domain, Part I: Posterior most probable value, Mechanical Systems and Signal Processing, 26 (2012) 60-75.

[42] S. Adhikari, Damping Models for Structural Vibration, Trinity College, University of Cambridge, Cambridge, England, 2000.

[43] R.W. Clough, J. Penzien, Dynamics of Structures, McGraw Hill, New York City, New York, 1975.

[44] W.K. HASTINGS, Monte Carlo sampling methods using Markov chains and their applications, Biometrika, 57 (1970) 97-109.

[45] G. Casella, E.I. George, Explaining the Gibbs Sampler, The American Statistician, 46 (1992) 167-174.

[46] R.M. Neal, Slice sampling, (2003) 705-767.

[47] Z. Zhang, C. Jiang, X. Han, D. Hu, S. Yu, A response surface approach for structural reliability analysis using evidence theory, Advances in Engineering Software, 69 (2014) 37-45.

[48] J.E. Hurtado, D.A. Alvarez, Neural-network-based reliability analysis: a comparative study, Computer Methods in Applied Mechanics and Engineering, 191 (2001) 113-132.

[49] C.M. Rocco, J.A. Moreno, Fast Monte Carlo reliability evaluation using support vector machine, Reliability Engineering \& System Safety, 76 (2002) 237-243.

[50] R.G. Ghanem, P. Spanos, Stochastic Finite Elements: A Spectral Approach, Springer, Berlin, Germany, 1991.

[51] V.J. Romero, L.P. Swiler, A.A. Giunta, Construction of response surfaces based on progressive-latticesampling experimental designs with application to uncertainty propagation, Structural Safety, 26 (2004) 201-219.

[52] S.S. Isukapalli, Uncertainty Analysis of Transport-Transformation, Rutgers, the State University of New Jersey, New Brunswick, New Jersey, 1999.

[53] S. Huang, S. Mahadevan, R. Rebba, Collocation-based stochastic finite element analysis for random field problems, Probabilistic Engineering Mechanics, 22 (2007) 194-205.

[54] G.A.F. Seber, C.J. Wild, Nonlinear Regression, John Wiley \& Sons, Inc., New York City, New York, 1989. 
[55] K. Christodoulou, C. Papadimitriou, Structural identification based on optimally weighted modal residuals, Mechanical Systems and Signal Processing, 21 (2007) 4-23.

[56] E.L. Zhang, P. Feissel, J. Antoni, A comprehensive Bayesian approach for model updating and quantification of modeling errors, Probabilistic Engineering Mechanics, 26 (2011) 550-560.

[57] E. Simoen, C. Papadimitriou, G. Lombaert, On prediction error correlation in Bayesian model updating, Journal of Sound and Vibration, 332 (2013) 4136-4152.

[58] Y. Ling, J. Mullins, S. Mahadevan, Selection of model discrepancy priors in Bayesian calibration, Journal of Computational Physics, 276 (2014) 665-680.

[59] G.B. Arhonditsis, D. Papantou, W. Zhang, G. Perhar, E. Massos, M. Shi, Bayesian calibration of mechanistic aquatic biogeochemical models and benefits for environmental management, Journal of Marine Systems, 73 (2008) 8-30.

[60] E.C. DeCarlo, S. Mahadevan, B.P. Smarslok, Bayesian Calibration of Aerothermal Models for Hypersonic Air Vehicles, 54th AIAA/ASME/ASCE/AHS/ASC Structures, Structural Dynamics, and Materials Conference, Boston, MA, 2013.

[61] S. Sankararaman, Y. Ling, C. Shantz, S. Mahadevan, Inference of equivalent initial flaw size under multiple sources of uncertainty, International Journal of Fatigue, 33 (2011) 75-89.

[62] R.G. Bower, I. Vernon, M. Goldstein, A.J. Benson, C.G. Lacey, C.M. Baugh, S. Cole, C.S. Frenk, The parameter space of galaxy formation, Monthly Notices of the Royal Astronomical Society, 407 (2010) 2017-2045.

[63] J. McFarland, S. Mahadevan, Multivariate significance testing and model calibration under uncertainty, Computer Methods in Applied Mechanics and Engineering, 197 (2008) 2467-2479.

[64] J.O. Berger, L.R. Pericchi, The Intrinsic Bayes Factor for Model Selection and Prediction, Journal of the American Statistical Association, 91 (1996) 109-122.

[65] X. Jiang, S. Mahadevan, Bayesian risk-based decision method for model validation under uncertainty, Reliability Engineering \& System Safety, 92 (2007) 707-718.

[66] T. Leonard, J.S.J. Hsu, Bayesian Methods: An Analysis for Statisticians and Interdisciplinary Researchers, Cambridge University Press, Cambridge, UK, 1999.

[67] H. Jeffreys, Theory of Probability, Oxford University Press Inc., Oxford, New York, 1961.

[68] A.G. Walshaw, Mechanical Vibration with applications, Ellis Horwood Ltd, New York, 1984.

[69] P.D. Welch, The use of fast Fourier transform for the estimation of power spectra: A method based on time averaging over short, modified periodograms, Audio and Electroacoustics, IEEE Transactions on, 15 (1967) 70-73. [70] R.E. Kass, L. Wasserman, The Selection of Prior Distributions by Formal Rules, Journal of the American Statistical Association, 91 (1996) 1343-1370. 
Figures caption:

Fig. 1: Half-power bandwidth method

Fig. 2: Actual and equivalent damping energy per cycle

Fig.3: Simple implementation of slice sampling

Fig. 4: Variation of $\varepsilon_{s u r}$ and $\varepsilon_{d}$ with model fidelity

Fig. 5: Curved panel dimensions and strain gage locations (units: $\mathrm{cm}$ )

Fig. 6: Boundaries with different fixity ratios $(F R):$ (a) $F R=0.5$, (b) $F R=1$

Table 1: Surrogate model properties

Table 2: Calibration parameters' prior distributions

Fig. 7: The model discrepancy posteriors - SG4. Prior $\sim N\left(\boldsymbol{\mu}_{\boldsymbol{d}}, \boldsymbol{\sigma}_{\boldsymbol{d}}\right)$

Fig. 8: Frictional damping posteriors. Prior $\sim$ Uniform $\left[-10^{-5}, 10^{-2}\right]$

Fig. 9: Material damping posteriors. Prior $\sim$ Uniform $\left[5.10^{-7}, 10^{-2}\right]$

Fig. 10: Fixity ratio posteriors. Prior Uniform $[0.7,1]$

Fig. 11: The model discrepancy posteriors for independent and correlated parameters

Fig. 12: The frictional damping posteriors for independent and correlated parameters

Fig. 13: The material damping posteriors for independent and correlated parameters

Fig. 14: The fixity ratio posteriors for independent and correlated parameters

Fig. 15: Preliminary model testing

Fig. 16: Time to convergence of three calibration strategies 